Article

\title{
Frequency Regulation System: A Deep Learning Identification, Type-3 Fuzzy Control and LMI Stability Analysis
}

\author{
Ayman A. Aly ${ }^{1}$, Bassem F. Felemban ${ }^{1}\left[\right.$, Ardashir Mohammadzadeh ${ }^{2, *}$, Oscar Castillo ${ }^{3}$ \\ and Andrzej Bartoszewicz 4 (D) \\ 1 Department of Mechanical Engineering, College of Engineering, Taif University, P.O. Box 11099, \\ Taif 21944, Saudi Arabia; aymanaly@tu.edu.sa (A.A.A.); b.felemban@tu.edu.sa (B.F.F.) \\ Independent Researcher, 1148 Baku, Azerbaijan \\ 3 Division of Graduate Studies and Research, Tijuana Institute of Technology, Tijuana 22414, Mexico; \\ ocastillo@tectijuana.mx \\ 4 Institute of Automatic Control, Lodz University of Technology, 18 Stefanowskiego St., 90-537 Łódź, Poland; \\ andrzej.bartoszewicz@p.lodz.pl \\ * Correspondence: a.mzadeh@ieee.org
}

Citation: Aly, A.A.; Felemban, B.F.; Mohammadzadeh, A.; Castillo, O.; Bartoszewicz, A. Frequency Regulation System: A Deep Learning Identification, Type-3 Fuzzy Control and LMI Stability Analysis. Energies 2021, 14, 7801. https://doi.org/ $10.3390 /$ en 14227801

Received: 21 October 2021

Accepted: 18 November 2021

Published: 22 November 2021

Publisher's Note: MDPI stays neutral with regard to jurisdictional claims in published maps and institutional affiliations.

Copyright: (c) 2021 by the authors. Licensee MDPI, Basel, Switzerland. This article is an open access article distributed under the terms and conditions of the Creative Commons Attribution (CC BY) license (https:// creativecommons.org/licenses/by/ $4.0 /)$.

\begin{abstract}
In this paper, the problem of frequency regulation in the multi-area power systems with demand response, energy storage system (ESS) and renewable energy generators is studied. Dissimilarly to most studies in this field, the dynamics of all units in all areas are considered to be unknown. Furthermore time-varying solar radiation, wind speed dynamics, multiple load changes, demand response (DR), and ESS are considered. A novel dynamic fractional-order model based on restricted Boltzmann machine (RBM) and deep learning contrastive divergence (CD) algorithm is presented for online identification. The controller is designed by the dynamic estimated model, error feedback controller and interval type-3 fuzzy logic compensator (IT3-FLC). The gains of error feedback controller and tuning rules of the estimated dynamic model are extracted through the fractional-order stability analysis by the linear matrix inequality (LMI) approach. The superiority of a schemed controller in contrast to the type- 1 and type-2 FLCs is demonstrated in various conditions, such as time-varying wind speed, solar radiation, multiple load changes, and perturbed dynamics.
\end{abstract}

Keywords: type-3 fuzzy systems; restricted Boltzmann machine; control systems; frequency regulation; linear matrix inequality

\section{Introduction}

By developing the renewable energy systems, the problem of the load frequency control (LFC) in power systems has become one of the interesting topics. Because the changes in solar radiation, time-varying wind speed, and variation of load power are the natural perturbation in these systems. The main controllers that frequently have been used are classified into 3 cases: simple PID controllers, fuzzy based PID controllers, and fractional-order PID controllers. The robust controllers with guaranteed stability are seldom investigated in literature [1-5].

In the first case, the conventional PID controller and lag-lead compensators are used. The main difference between studies is the type of optimization algorithm. For instance, in [6], the imperialist competitive optimization technique is proposed to adjust the coefficients of PID control system and its regulation function is compared by the PI controller optimized with genetic algorithm. In [7], the optimized PID controller by a chaotic optimization method is proposed and its effectiveness is shown by applying on two areas power system. In [8], the capability of the genetic algorithm is examined on the PID frequency regulation problem. In [9], the grey wolf optimization method is developed for designing a PID control scheme for LFC. In [10], honey bee mating method is utilized for obtaining the optimized gains for PID and its regulation proficiency is investigated. 
The PID controller tuned by bacterial foraging method is designed in [11] for two areas system and its efficiency is studied in contrast to the conventional PID and optimized PID by genetic algorithm. In [12], the PID controller is tuned on basis of grey wolf method and it is shown that the regulation performance is progressed in term of peak overshoot.

In the second case, the fuzzy PID (FPID) controllers have been developed for LFC. The fuzzy systems are one of the best approach to deal with the uncertainties [13-15]. In this case, commonly the type- 1 fuzzy logic systems (T1-FLSs) are employed to extract the gains of the PID control scheme and various optimization techniques specially evolutionary based algorithms are used for optimization of the parameters of FLSs. For example, in [16], a FPID is designed and the big bang-big crunch algorithm is proposed for optimization. In [17], the fuzzy PI controller is generated using the bat algorithm for LFC, and its superiority is evaluated in comparison to other similar controllers, such as conventional PID. In [18], the teaching-learning optimization algorithm is studied for designing the FPID controller for LFC and it is verified that the regulation performance is improved in contrast to the other optimized PID controllers. In [19], the cuckoo and harmony search algorithms are suggested for designing the FPID controller for LFC. In [20], the multi-verse algorithm is proposed for optimization of the FPID and its proficiency is evaluated by applying on two-area power system. The differential evolution tuning technique is developed in [21] for optimizing the FPID controller. In [22], the robustness of the FPID controller is studied on two areas of the photovoltaic-thermal system. In [23], the sine-cosine algorithm is used for designing FPID. In [24], the effectiveness of grey wolf algorithm is studied in comparison with the bee colony method in the designing of FPID controller for LFC. In [25], the improvement of frequency regulation of FPID controller based on the moth swarm optimization approach is studied by providing an real-time examination.

For the third case, recently the fractional-order (FO) PID (FO-PID) control technique and FO fuzzy logic controllers (FLCs) are also developed for LFC [26,27]. For example, in [28], the FO-PID controller is designed on the basis of a symbiotic organisms technique and is applied for frequency control in a microgrid. In [29], similarly to the previously reviewed studies, the FO-PID controller is optimized by multi-verse method and its superiority is authenticated by several simulations. In [30], the frequency regulation performance of the traditional PID is improved employing fractional-order calculus and moth flame tuning method. In [31], the effectiveness of the FO control scheme is examined by applying on single and two areas system. In [32], the FO-PID controller is improved by Salp swarm optimization algorithm and time-delay effect is analyzed. In [33], the impact of some time-varying factors in the dynamics of a two areas power system is taken into account and a FO-PID is designed and it is authenticated that FO-PID is more effective.

In most of the above reviewed studies, the closed-loop stability is not investigated and also the control system is presented for a special power system with restricted objects and areas. Furthermore, the evolutionary based optimization algorithms are utilized to adjust the control parameters that impose a high computational cost beside of lack of stability guarantee. Additionally, the effect of natural disturbances, such as simultaneous changes of the wind speed, solar radiation, and load perturbation, are rarely studied. The robustness of the controller are quite seldom studied. For example in [34], the event triggered controller is combined with the $H_{\infty}$ criteria to ensure the robustness of the frequency regulator. Considering the aforementioned literature review and investigation, in this study a novel intelligent frequency regulator is suggested for multi-areas power system. Dissimilarly to the reviewed studies, the system model is online identified by the suggested dynamic fractional-order RBM. A new compensator is designed by the IT3-FLS to tackle the effect of the approximation error and other perturbations. The closed-loop stability is analysed by LMI approach. The main contributions of this study are:

- The dynamics of all units in all areas are considered to be unknown;

- In addition to unknown dynamics, the effects of time-varying parameters, solar radiation, wind speed, and multiple load changes are taken to account; 
- A novel dynamic fractional-order model using RBMs and deep learning algorithm is proposed for online identification;

- A novel compensator on basis of IT3-FLS is presented to cope with the dynamic model approximation error;

- A new LMI approach is developed to derive the error feedback gains and to guarantee the stability and robustness.

In the remain of this study, the problem is described in Section 3, the suggested dynamic fractional-order model is described in Section 4, the proposed IT3-FLC is given in Section 5, the stability is analyzed Section 6, the simulations and conclusions are presented in Sections 7 and 8, respectively.

\section{System Description}

The under control plant is shown in Figure 1. The case study plant, includes, loads, photovoltaic (PVs), battery/flywheel ESS (BESS)/(FESS), micro-turbines, and wind turbine (WT). Consider the $k$-th area, the power changing is written as $[35,36]$ :

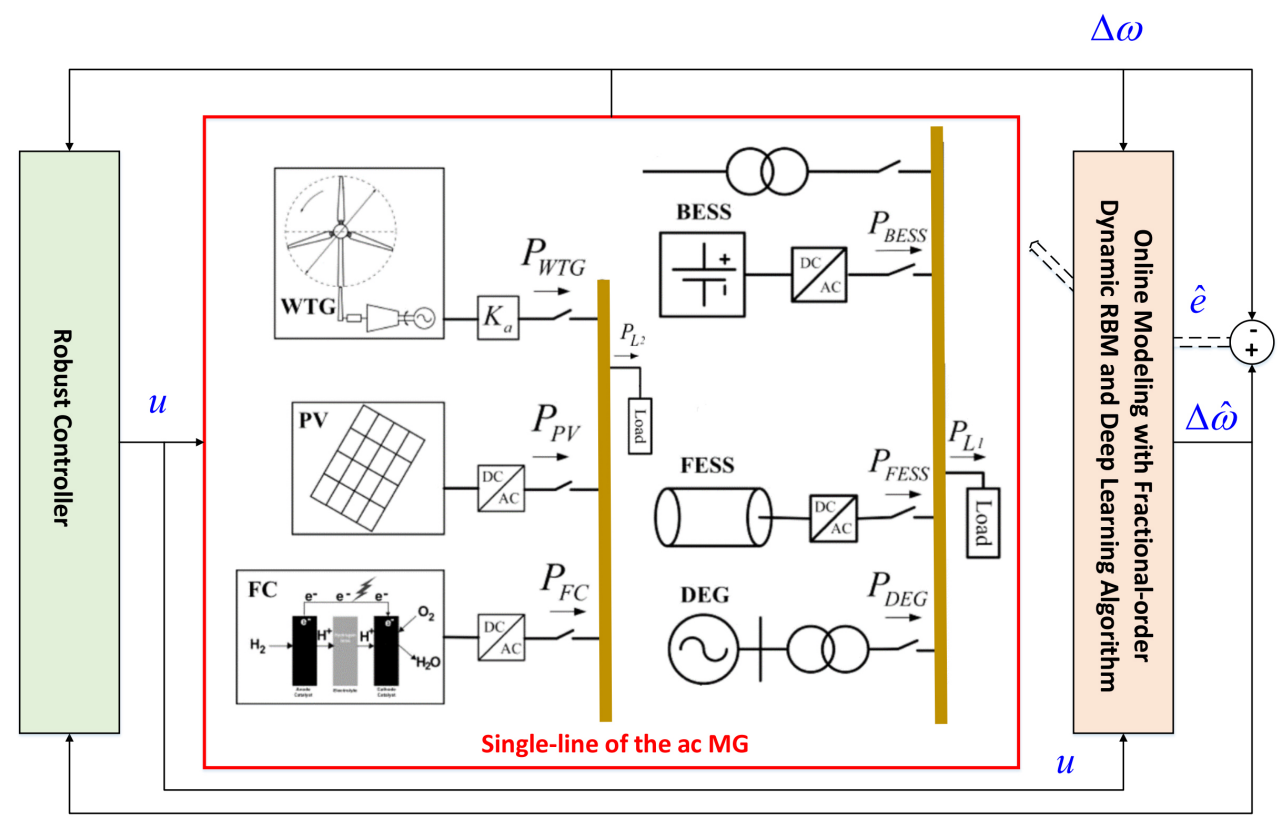

Figure 1. The suggested control block diagram.

$$
\begin{aligned}
\Delta P_{k, t i e} & =\sum_{i=1, i \neq k}^{N} \Delta P_{k i, t i e} \\
& =\frac{2 \pi}{s}\left[\sum_{i=1, i \neq k}^{N} T_{k i} \Delta \omega_{k}-\sum_{i=1, i \neq k}^{N} T_{k i} \Delta \omega_{i}\right]
\end{aligned}
$$

where, $N$ represents number of area, $\Delta P_{k, t i e}$ represents the power variation between tie-line and $k$-th area, $\Delta \omega$ is frequency deviation and $T_{k i}$ is torque coefficient of tie-line synchronization. Consider a disturbance applied to 1-th area as:

$$
\Delta P_{1, t i e}=\frac{2 \pi}{s}\left[T_{12} \Delta \omega_{1}-T_{12} \Delta \omega_{2}\right]
$$

Then, $\Delta \omega_{2}$ is:

$$
\Delta \omega_{2}=\frac{\left(-\Delta P_{2, t i e}-\Delta P_{2 L}+\sum_{k=1}^{n} \Delta P_{m 2 i}\right)}{D_{2}+2 H_{2} s}
$$


Then, by applying the load disturbance into $k$-th area, $\Delta P_{k, t i e}$ and $\Delta \omega_{k}$ are written as:

$$
\begin{gathered}
\Delta P_{k, t i e}=\frac{2 \pi}{s}\left[\sum_{i=1, i \neq k}^{N} T_{k i} \Delta \omega_{k}\right] \\
\Delta \omega_{k}=\frac{\left(-\Delta P_{k, t i e}-\Delta P_{k L}\right)}{D_{k}+2 H_{k} s}
\end{gathered}
$$

Considering a step perturbation in load as $\Delta P_{k L}(s)=\Delta P_{k L} / s$ and from (5), $\Delta P_{k, t i e}$ becomes:

$$
\Delta P_{k, t i e}=\frac{-2 \pi \Delta P_{k L} \sum_{i=1, i \neq k}^{N} T_{k i} / 2 H_{k}}{\left(s^{2}+D_{k} / 2 H_{k}+2 \pi \sum_{i=1, i \neq k}^{N} T_{k i} / 2 H_{k}\right) s}
$$

The regional demand response (RDR) is implemented as follows. If $\frac{d^{2} \Delta P_{k, t i e}}{d t^{2}}<0$ and $\frac{d^{2} \Delta P_{i, t i e}}{d t^{2}}>0$ or $\frac{d^{2} \Delta P_{k, \text { tie }}}{d t^{2}}>0$ and $\frac{d^{2} \Delta P_{i, t i e}}{d t^{2}}<0$, then:

$$
R D R_{k}=\delta \frac{-\frac{d^{2} \Delta P_{k, t i e}}{d t^{2}} H_{k}}{\pi \sum_{i=1, i \neq k}^{N} T_{k i}}
$$

where, $0<\delta<1$. The dynamics of the other units are given in Figure 2 .

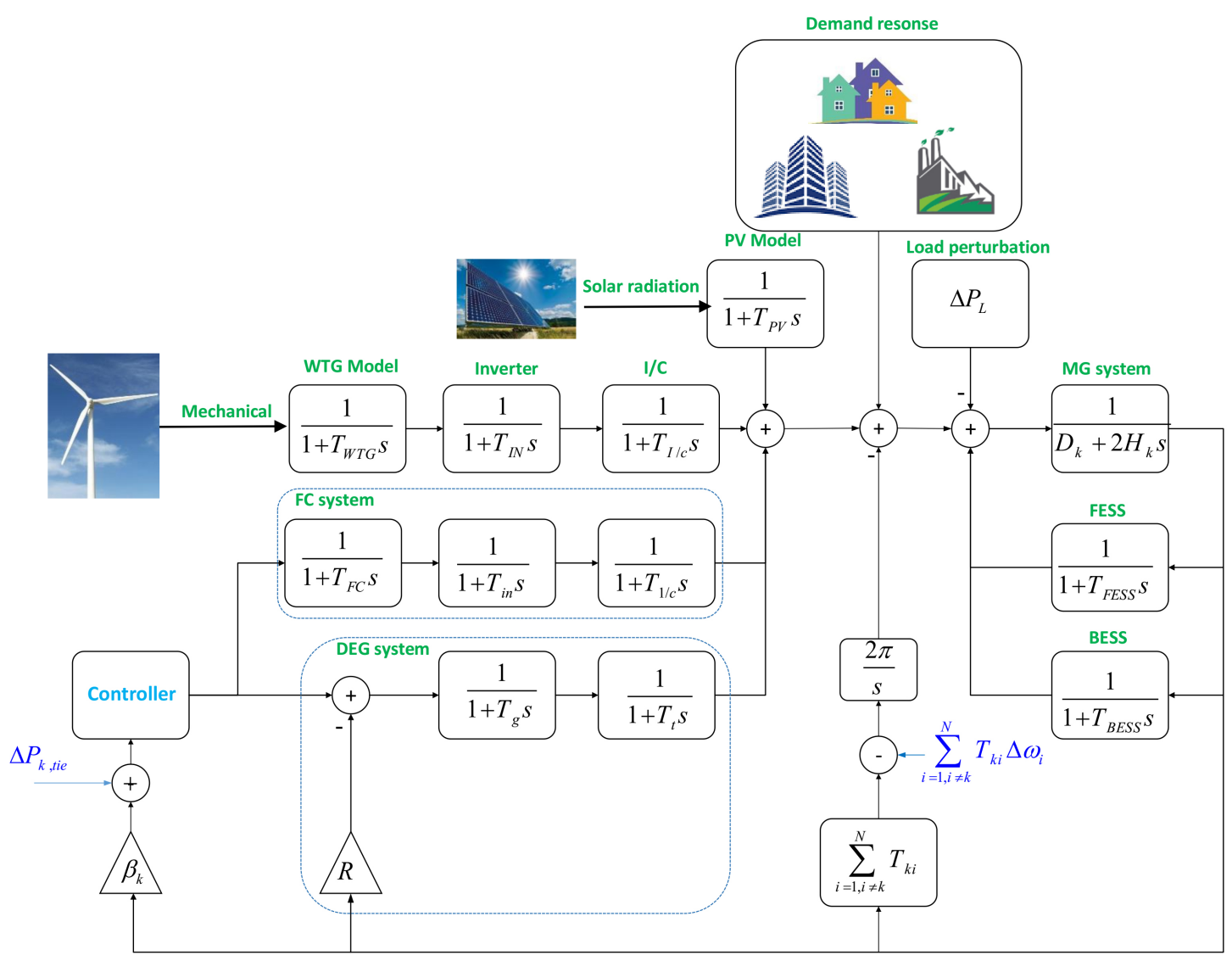

Figure 2. The general diagram of case study. 


\section{A General View on Presented Control System}

The general block diagram is depicted in Figure 3. Unlike in most of the studies, the dynamics of all units are assumed to be completely unknown. The dynamics of all units are estimated by the proposed dynamic RMB and CD deep learning algorithm. It should be noted that the estimated dynamic model is online optimized then it can handle the system dynamic perturbation. The dynamic model is:

$$
D_{t}^{q} \Delta \hat{\omega}_{k}=\hat{f}_{k}\left(x_{k} \mid \theta_{k m}, \tilde{\xi}_{k}, \varepsilon_{k}, \eta_{k}\right)+\chi_{k} \Delta \tilde{\omega}_{k}+u_{k}
$$

where, $\hat{f}_{k}$ is the proposed RBMs, $u_{k}$ is the control signal, $\xi_{k}, \varepsilon_{k}$, and $\eta_{k}$ are the parameters of RBM, $\theta_{k m}$ is the vector of parameters of MLP, $x_{k}$ is the vector of inputs of $\hat{f}_{k}$ that is shown in Figure $3, \chi_{k}$ is a positive constant that is obtained form stability analysis, $\Delta \tilde{\omega}_{k}=\Delta \omega_{k}-\Delta \hat{\omega}_{k}$, $\omega_{k}$ is the frequency deviation and $D_{t}^{q} \Delta \omega_{k}$ is defined as:

$$
D_{t}^{q} \Delta \omega_{k}(t)=1 / \Gamma(m-q) \int_{0}^{t} \frac{1}{(t-\tau)^{m-q-1}} \frac{d^{m}}{d t^{m}} \Delta \omega_{k}(\tau) d \tau
$$

$\Gamma(\cdot)$ represents Gamma function, $0<q<1$ and $m=1$. For each area the controller is designed as:

$$
u_{k}=-\hat{f}_{k}-\lambda_{k} \Delta \omega_{k}-u_{c k}\left(x_{c k} \mid \theta_{c}\right)
$$

where, $u_{c k}$ is the compensator that is generated by IT3-FLS, $\lambda_{k}$ is determined by LMI stability analysis. $\theta_{c}$ is the vector of parameters of $u_{c k}$ and $x_{c k}$ is the vector of inputs of $u_{c k}$. The parameters of $u_{c k}$ are learned by error feedback method.

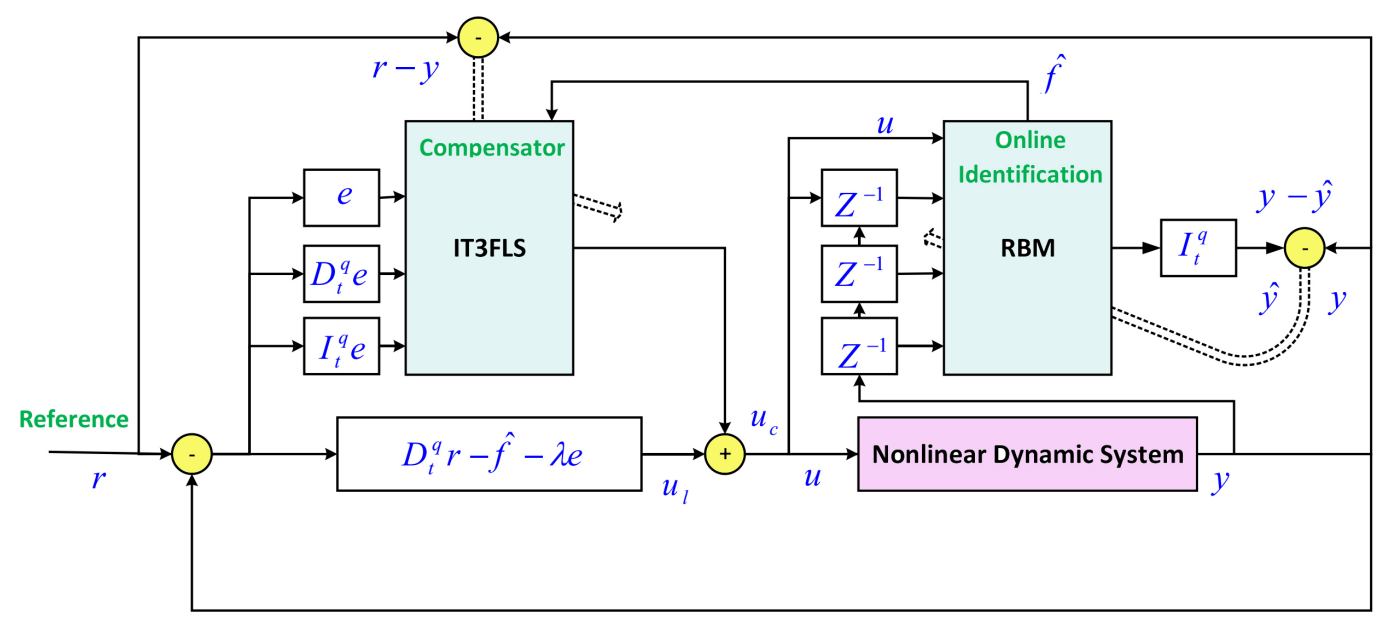

Figure 3. The suggested control block diagram.

\section{Proposed Dynamic Fractional-Order Model}

\subsection{Structure}

The structure of the proposed dynamic fractional-order RMB-MLP is depicted in Figure 4 . The parameters of RBM are tuned by $\mathrm{CD}$ algorithm and the vector of parameters $\theta_{m}$ is adjusted by the tuning rule that is derived through the LMI based stability analysis. Consider $k$-th RBM-MLP, the output of proposed dynamic model is computed as follows:

(1) The inputs of RBM-MLP are the outputs of the system at the previous sample times;

(2) The inputs are considered as the visible nodes $V_{k} 0$ and the outputs in the hidden layer $H_{k} 0$ are obtained as:

$$
H_{k 0}=\Omega\left(\xi_{k}^{T} V_{k 0}+\varepsilon_{k}\right)
$$

where, $\xi_{k}$ is a $\tau \times n$ matrix, $\tau$ is the number of inputs, $n$ is the number of neurons, $\varepsilon_{k}$ is $n \times 1$ vector and $\Omega(\cdot)=1 /(1+\exp (\cdot))$; 
(3) From $H_{k 0}$, the vector $V_{k 1}$ is obtained as:

$$
V_{k 1}=\Omega\left(\xi_{k} H_{k 0}+\eta_{k}\right)
$$

where, $H_{k 0}$ that is obtained in the previous step is $n \times 1$ vector and $\eta_{k}$ is a $\tau \times 1$ vector;

(4) Similarly form $V_{k 1}$ the vector $H_{k 1}$ is obtained as:

$$
H_{k 1}=\Omega\left(\xi_{k}^{T} V_{k 1}+\varepsilon_{k}\right)
$$

where, $H_{k 1}$ is a $n \times 1$ vector;

(5) Finally the output is obtained as:

$$
\Delta \hat{\omega}_{k}=I_{t}^{q}\left(\theta_{m k}^{T} H_{k 1}+u_{k}\right)
$$

where, $I_{t}^{q}$ represents the fractional integral, $u_{k}$ is the control signal in the $k$-th area and $\theta_{m k}$ is $n \times 1$ vector.

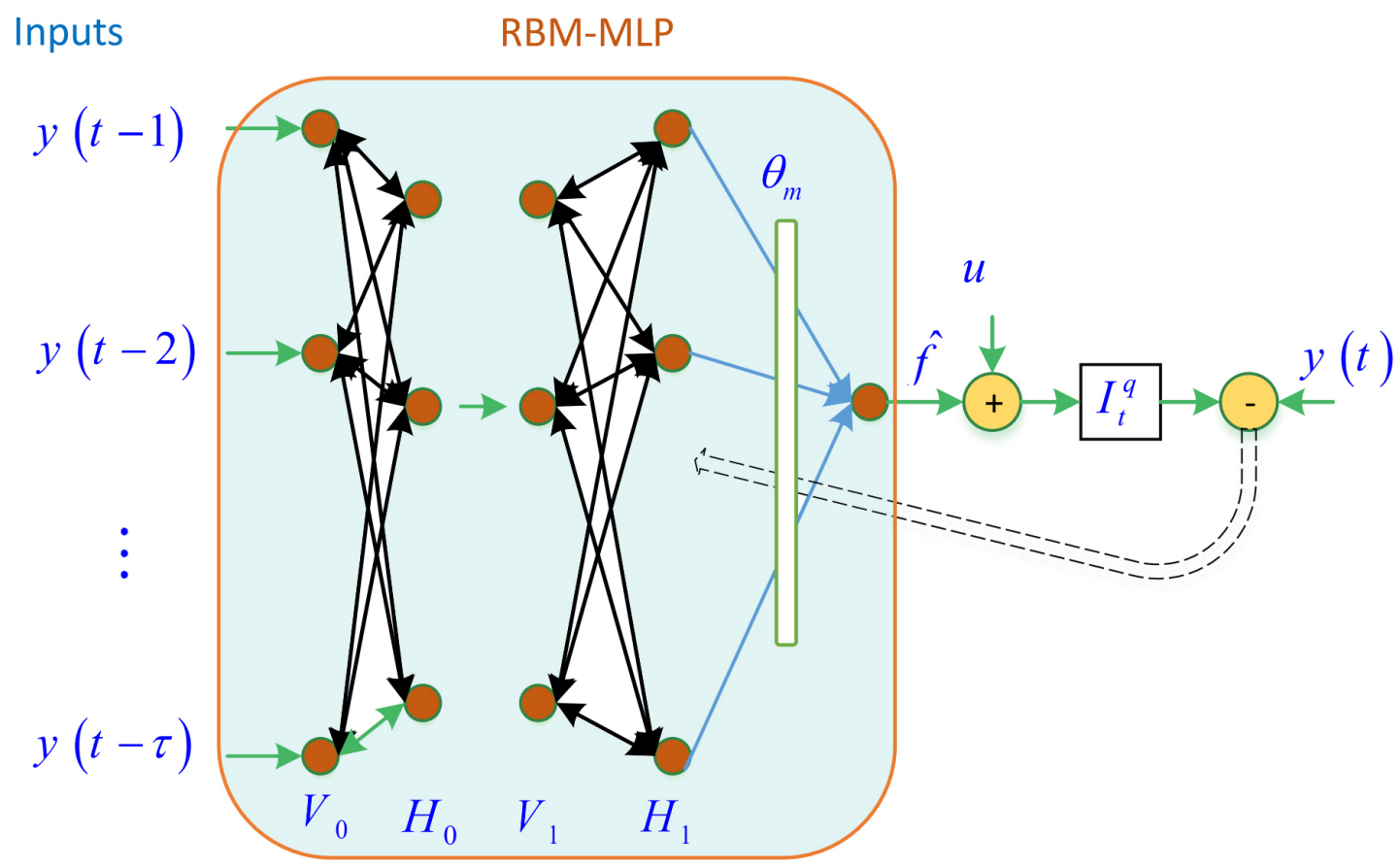

Figure 4. The suggested fractional-order dynamic RBM-MLP block diagram.

\subsection{Learning}

The adjusting of vector $\theta_{m k}^{T}$ is completed by the tuning rule (42) and the learning of parameters $\xi_{k}, \varepsilon_{k}$ and $\eta_{k}$ are performed by CD algorithm, in which, the probability and energy function are defined as follows:

$$
\begin{gathered}
\rho(\xi, \varepsilon, \eta, V, H)=e^{\mathrm{E}(\xi, \varepsilon, \eta, V, H)} / \mathrm{E}(\xi, \varepsilon, \eta, V, H) \\
\mathrm{E}(\xi, \varepsilon, \eta, V, H)=-H^{T} \xi V-\varepsilon^{T} H-\eta^{T} V
\end{gathered}
$$

Then the tuning rules are considered as:

$$
\begin{gathered}
\xi_{k}(t+1)=\xi_{k}(t)+\gamma\left(V_{k 0} H_{k 0}^{T}-V_{k 1} H_{k 1}^{T}\right) \\
\varepsilon_{k}(t+1)=\varepsilon_{k}(t)+\gamma\left(H_{k 0}-H_{k 1}\right)
\end{gathered}
$$




$$
\eta_{k}(t+1)=\eta_{k}(t)+\gamma\left(V_{k 0}-V_{k 1}\right)
$$

\section{Proposed Type-3 Fuzzy Compensator}

\subsection{Structure}

The suggested compensator is an IT3FLS. The structure is given in Figure 5. The output of compensator is obtained as:

(1) The inputs are $e(t), D_{t}^{q} e(t)$ and $I_{t}^{q} e(t)$, where, $e(t) D_{t}^{q} e(t)$ and $I_{t}^{q} e(t)$ represent the stabilization error, fractional derivative and integral of stabilization error, respectively;

(2) For each inputs $x c_{1}=e(t), x c_{2}=D_{t}^{q} e(t)$ and $x c_{3}=I_{t}^{q} e(t), R$ membership functions (MFs) are considered as $\tilde{A}_{s}^{l} \mid \alpha_{\iota}, s=1,2,3, l=1, \ldots, R$ with $r$ slices $(\iota=1, \ldots, r)$. Then for input $x c_{s}$, one has:

$$
\begin{aligned}
& \bar{\mu}_{\tilde{A}_{s}^{l} \mid \bar{\alpha}_{t}}\left(x c_{s}\right)=\exp \left(-\frac{\left(x_{s}-c_{\tilde{A}_{s}^{l}}\right)^{2}}{\bar{\sigma}_{\tilde{A}_{s}^{l} \mid \bar{\alpha}_{t}}^{2}}\right) \\
& \bar{\mu}_{\tilde{A}_{s}^{l} \mid \underline{\alpha}_{t}}\left(x c_{s}\right)=\exp \left(-\frac{\left(x_{s}-c_{\tilde{A}_{s}^{l}}\right)^{2}}{\bar{\sigma}_{\tilde{A}_{s}^{l} \mid \underline{\alpha}_{t}}^{2}}\right) \\
& \underline{\mu}_{\tilde{A}_{s}^{l} \mid \bar{\alpha}_{t}}\left(x c_{s}\right)=\exp \left(-\frac{\left(x_{s}-c_{\tilde{A}_{s}^{l}}\right)^{2}}{\underline{\sigma}_{\tilde{A}_{s}^{l} \mid \bar{\alpha}_{\iota}}^{2}}\right) \\
& \underline{\mu}_{\tilde{A}_{s}^{l} \mid \underline{\alpha}_{t}}\left(x c_{s}\right)=\exp \left(-\frac{\left(x_{s}-c_{\tilde{A}_{s}^{l}}\right)^{2}}{\underline{\sigma}_{\tilde{A}_{s}^{l} \mid \underline{\alpha}_{t}}^{2}}\right)
\end{aligned}
$$

where, $c_{\tilde{A}_{s}^{l}}$ is the center of Gaussian MF $\tilde{A}_{s}^{l}, \bar{\sigma}_{\tilde{A}_{s}^{l} \mid \bar{\alpha}_{l}}$ and $\bar{\sigma}_{\tilde{A}_{l}^{l} \mid \underline{\alpha}_{l}}$ are the upper standard division of $\tilde{A}_{s}^{l}$ at $\bar{\alpha}_{\iota}$ and $\underline{\alpha}_{\iota}$ levels, respectively, and $\underline{\sigma}_{\tilde{A}_{s}^{l} \mid \bar{\alpha}_{l}}$ and $\underline{\sigma}_{\tilde{A}_{s}^{l} \mid \alpha_{l}}$ are the lower standard division of $\tilde{A}_{s}^{l}$ at slice level $\bar{\alpha}_{l}$ and $\underline{\alpha}_{l}$, respectively (see Figure 6);

(3) For each MFs in the step 2, one rule is considered. Then the number of rules is $R$ and rule firings are:

$$
\begin{aligned}
& \bar{z}_{\bar{\alpha}_{l}}^{l}=\bar{\mu}_{\tilde{A}_{1}^{l} \mid \bar{\alpha}_{t}}(e) \bar{\mu}_{\tilde{A}_{2}^{l} \mid \bar{\alpha}_{t}}\left(D_{t}^{q} e\right) \bar{\mu}_{\tilde{A}_{3}^{l} \mid \bar{\alpha}_{t}}\left(I_{t}^{q} e\right) \\
& \bar{z}_{\underline{\alpha}_{t}}^{l}=\bar{\mu}_{\tilde{A}_{1}^{l} \mid \underline{\alpha}_{l}}(e) \bar{\mu}_{\tilde{A}_{1}^{l} \mid \underline{\alpha}_{t}}\left(D_{t}^{q} e\right) \bar{\mu}_{\tilde{A}_{1}^{l} \mid \underline{\alpha}_{t}}\left(I_{t}^{q} e\right) \\
& \underline{z}_{\bar{\alpha}_{l}}^{l}=\underline{\mu}_{\tilde{A}_{1}^{l} \mid \bar{\alpha}_{t}}(e) \underline{\mu}_{\tilde{A}_{2}^{l} \mid \bar{\alpha}_{t}}\left(D_{t}^{q} e\right) \underline{\mu}_{\tilde{A}_{3}^{l} \mid \bar{\alpha}_{t}}\left(I_{t}^{q} e\right) \\
& \underline{z}_{\underline{\alpha}_{l}}^{l}=\underline{\mu}_{\tilde{A}_{1}^{l} \mid \underline{\alpha}_{l}}(e) \underline{\mu}_{\tilde{A}_{1}^{l} \mid \underline{\alpha}_{l}}\left(D_{t}^{q} e\right) \underline{\mu}_{\tilde{A}_{1}^{l} \mid \underline{\alpha}_{t}}\left(I_{t}^{q} e\right)
\end{aligned}
$$

(4) By type-reduction in [37], the output of compensator is concluded as:

$$
u_{c}=\frac{\sum_{l=1}^{r} \bar{\alpha}_{\iota} \bar{\nu}_{\iota}+\underline{\alpha}_{\iota} \underline{\nu}_{l}}{\sum_{l=1}^{r}\left(\bar{\alpha}_{\iota}+\underline{\alpha}_{l}\right)} e
$$

where,

$$
\bar{v}_{\iota}=\frac{\sum_{l=1}^{R}\left(\bar{z}_{\bar{\alpha}_{l}}^{l}+\underline{z}_{\bar{\alpha}_{l}}^{l}\right) \bar{\theta}_{c l}}{\sum_{l=1}^{R}\left(\bar{z}_{\bar{\alpha}_{l}}^{l}+\underline{z}_{\bar{\alpha}_{l}}^{l}\right)}
$$




$$
\underline{v}_{l}=\frac{\sum_{l=1}^{R}\left(\bar{z}_{\underline{\alpha}_{l}}^{l}+\underline{z}_{\underline{\alpha}_{l}}^{l}\right) \underline{\theta}_{c l}}{\sum_{l=1}^{R}\left(\bar{z}_{\underline{\alpha}_{l}}^{l}+\underline{z}_{\underline{\alpha}_{l}}^{l}\right)}
$$

where, $\bar{\theta}_{l}$ and $\underline{\theta}_{c l}$ are the upper and lower $l$-th rule parameters. The vector of rule parameters is:

$$
\theta_{c}=\left[\underline{\theta}_{c 1}, \ldots, \underline{\theta}_{c R}, \bar{\theta}_{c 1}, \ldots, \bar{\theta}_{c R}\right]^{T}
$$

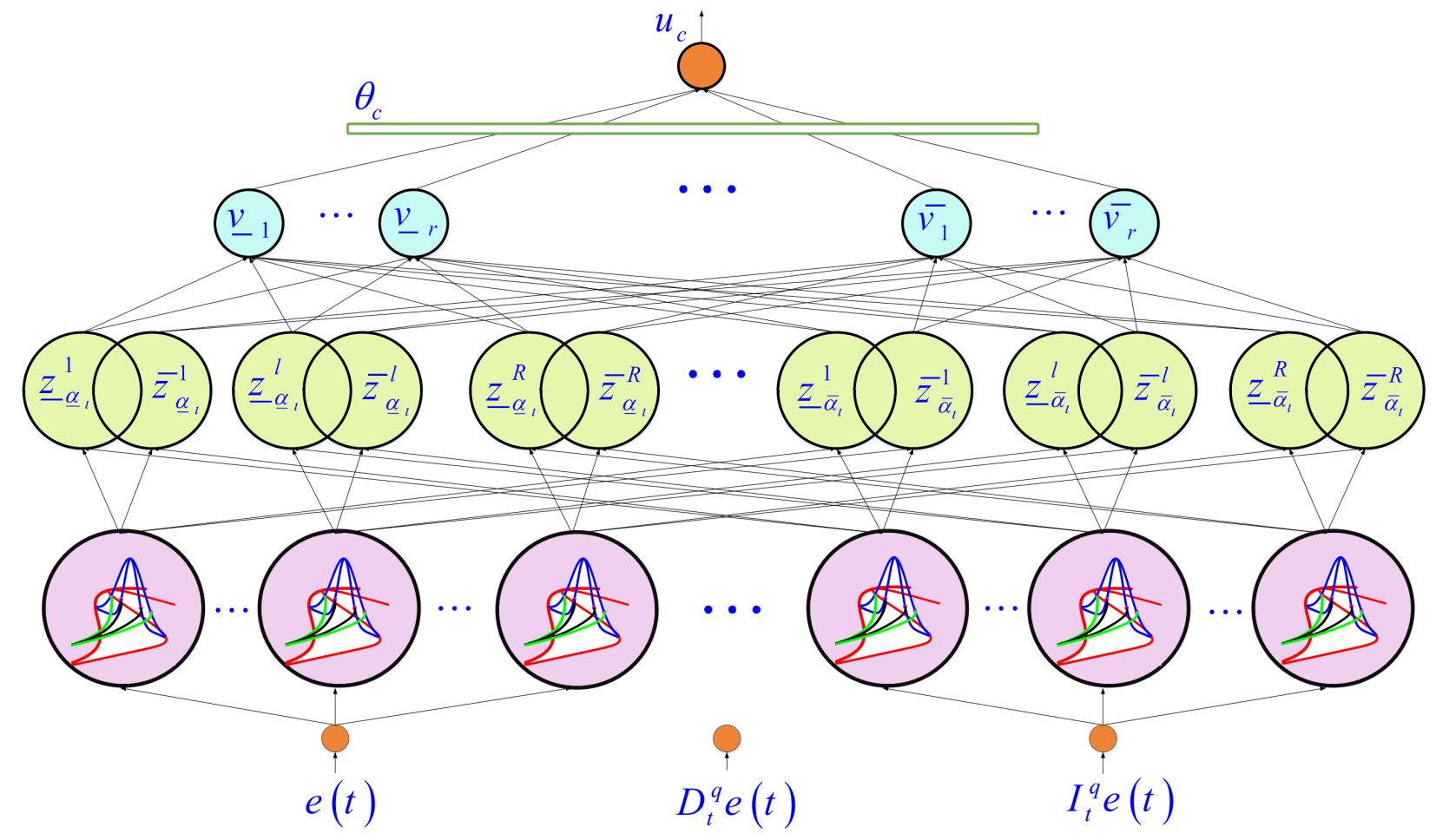

Figure 5. The suggested interval type-3 FLS block diagram.

\subsection{Learning}

The parameters of compensator are tuned by the simple gradient descent algorithm, such that the cost function $J=\frac{1}{2} e^{2}(t)$ is minimized:

$$
\begin{aligned}
& \bar{\theta}_{c l}(t+1)=\bar{\theta}_{c l}(t)-\gamma \frac{\partial J}{\partial \bar{\theta}_{c l}} \\
& \underline{\theta}_{c l}(t+1)=\underline{\theta}_{c l}(t)-\gamma \frac{\partial J}{\partial \underline{\theta}_{c l}}
\end{aligned}
$$

where,

$$
\begin{gathered}
\frac{\partial J}{\partial \bar{\theta}_{c l}}=\frac{e^{2}}{\sum_{l=1}^{r}\left(\bar{\alpha}_{l}+\underline{\alpha}_{l}\right)} \sum_{l=1}^{r} \frac{\bar{\alpha}_{l}\left(\bar{z}_{\bar{\alpha}_{\iota}}^{l}+\underline{z}_{\bar{\alpha}_{l}}^{l}\right)}{\sum_{l=1}^{R}\left(\bar{z}_{\bar{\alpha}_{l}}^{l}+\underline{z}_{\bar{\alpha}_{l}}^{l}\right)} \\
\frac{\partial J}{\partial \underline{\theta}_{c l}}=\frac{e^{2}}{\sum_{l=1}^{r}\left(\bar{\alpha}_{\iota}+\underline{\alpha}_{l}\right)} \sum_{l=1}^{r} \frac{\underline{\alpha}_{l}\left(\bar{z}_{\underline{\alpha}_{l}}^{l}+\underline{z}_{\underline{\alpha}_{l}}^{l}\right)}{\sum_{l=1}^{R}\left(\bar{z}_{\underline{\alpha}_{l}}^{l}+\underline{z}_{\underline{\alpha}_{l}}^{l}\right)}
\end{gathered}
$$




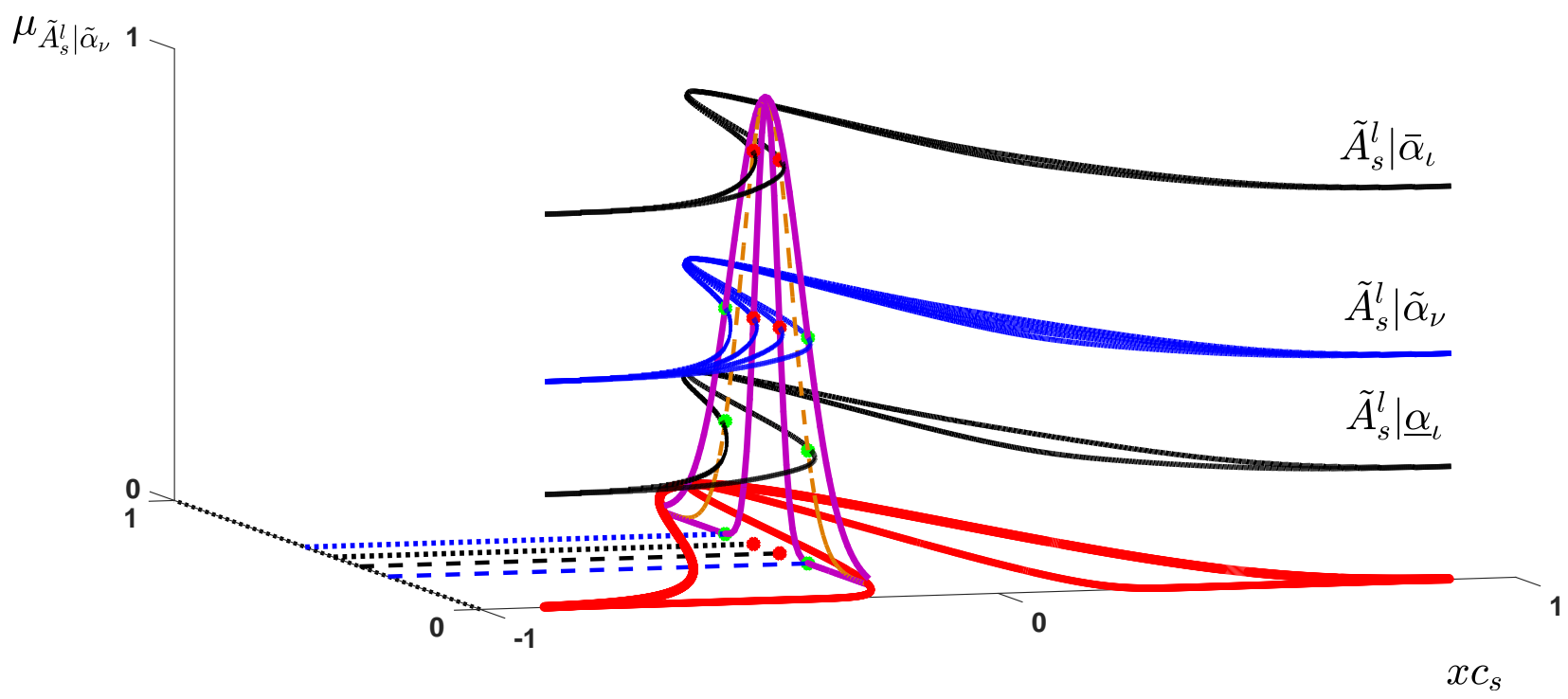

Figure 6. Type-3 MF.

It should be noted that the sign of derivative of output $(\Delta \omega)$ with respect to the control signal is positive and it is fixed. Then, the value of $\frac{\partial \Delta \omega}{\partial u_{c}}$ is combined with $\gamma$.

\section{Stability Analysis}

The main stability outcomes are presented in the following theorem:

Theorem 1. The asymptotic stability of closed-loop system shown in Figure 2, is ensured, if the tuning rule and control signal are as (40)-(42) and there are scalars $Q_{1}, Q_{2}, X_{1}, X_{2}, \vartheta_{1}, \vartheta_{2}$ and symmetric matrix $Q_{3} \in R^{n \times n}$, such that:

$$
\left[\begin{array}{ll}
\Psi_{11} & \Psi_{12} \\
\Psi_{12} & \Psi_{22}
\end{array}\right]<0
$$

where,

$$
\begin{aligned}
& \Psi_{11}=\sum_{i=1}^{2} \operatorname{Sym}\left\{\Theta_{i 1} \otimes\left[\begin{array}{ccc}
-X_{k 1}-\varphi_{c k}^{T} \theta_{c k} Q_{1} & X_{k 2} & \underline{0}_{1 \times n} \\
0 & -X_{k 2} & H_{k 1}^{T} Q_{3} \\
\underline{0}_{n \times 1} & -\gamma H_{k 1} Q_{2} & \underline{0}_{n \times n}
\end{array}\right]\right\}+ \\
& \sum_{i=1}^{2} \vartheta_{i}\left(I_{2} \otimes\left[\begin{array}{ccc}
0 & 0 & \underline{0}_{1 \times n} \\
\bar{E}_{k} \bar{E}_{k}^{T} & 0 & \underline{0}_{1 \times n} \\
\underline{0}_{n \times 1} & \underline{0}_{n \times 1} & \underline{0}_{n \times n}
\end{array}\right]\right) \\
& \Psi_{12}=\left[\left(I_{(n+2)} \otimes\left[\begin{array}{ccc}
Q_{1} & 0 & \underline{0}_{1 \times n} \\
0 & Q_{1} & \underline{0}_{1 \times n} \\
\underline{0}_{n \times 1} & \underline{0}_{n \times 1} & \underline{0}_{n \times n}
\end{array}\right]\right)^{T}\left(I_{(n+2)} \otimes\left[\begin{array}{ccc}
Q_{1} & 0 & \underline{0}_{1 \times n} \\
0 & Q_{1} & \underline{0}_{1 \times n} \\
\underline{0}_{n \times 1} & \underline{0}_{n \times 1} & \underline{0}_{n \times n}
\end{array}\right]\right)\right] \\
& \Psi_{22}=\left[\begin{array}{cc}
-\vartheta_{1} & 0 \\
0 & -\vartheta_{2}
\end{array}\right] \otimes I_{2} \\
& u_{k}=-\hat{f}_{k}-\lambda_{k} \Delta \omega_{k}-u_{c k}\left(x_{c k} \mid \theta_{c k}\right) \\
& u_{c k}\left(x_{c k} \mid \theta_{c k}\right)=-\varphi_{c k}^{T} \theta_{c k} \Delta \hat{\omega}_{k} \\
& D_{t}^{q} \theta_{m}=-\gamma H_{k 1} \Delta \tilde{\omega}_{k}
\end{aligned}
$$

where, $u_{c k}\left(x_{c k} \mid \theta_{c k}\right)$ is the compensator.

Proof. The proof is given in Appendix A. 


\section{Simulation}

The regulation outcomes of the designed LFC is examined in several scenarios, such as time-varying dynamics, suddenly changes in mechanical power, time-varying solar radiation, multiple load perturbation, and so on.

Example 1. For the first example an AC microgrid with given parameters as Table 1 is considered. For each inputs of IT3-FLS 3 MFs with centers $-1,1$ and 1 are considered. The other control parameters are presented in Table 2. The performance is evaluated in four scenarios.

Table 1. System parameter description.

\begin{tabular}{ccccccc}
\hline Parameters & Value/Unit & Parameters & Value/Unit & Unit & Power (KW) & Output Load (KW) \\
\hline$T_{B E S S}$ & $0.1(\mathrm{~s})$ & $T_{g}$ & $0.08(\mathrm{~s})$ & DEG & 160 & $P_{L_{2}}=$ \\
\hline$T_{P V}$ & $1.80(\mathrm{~s})$ & $T_{W T G}$ & $1.50(\mathrm{~s})$ & PV & 31 & $P_{L_{1}}=$ \\
\hline $\mathrm{H}$ & $0.17(\mathrm{pu} \mathrm{s})$ & $T_{F E S S}$ & $0.1(\mathrm{~s})$ & FC & 70 & \\
\hline$T_{t}$ & $0.4(\mathrm{~s})$ & $T_{I / c}$ & $0.0040(\mathrm{~s})$ & FESS & 46 & 210 \\
\hline$T_{I N}$ & $0.040(\mathrm{~s})$ & $R$ & $0.330(\mathrm{pu} / \mathrm{Hz})$ & BESS & 45 & \\
\hline
\end{tabular}

Table 2. Control parameters.

\begin{tabular}{ccc}
\hline Parameter & Value & Reference \\
\hline$q$ & 0.5 & $(8)$ \\
$\lambda$ & 100 & $(40)$ \\
$r$ & 3 & $(28)$ \\
$\alpha$ & $0,0.5,1$ & $(28)$ \\
$\gamma$ & 0.1 & $(17)-(19)$ \\
$n$ & 10 & $(11)$ \\
\hline
\end{tabular}

Scenario 1: For the first scenario, the parameters are fixed and one load change $\left(\Delta p_{L}\right)$ is considered. The other parameters and factors, such as solar radiation and wind speed, are also considered to be fixed. The regulation performance is depicted in Figure 7. The performance is compared with the some similar popular controllers, such as PI type-1 FLC (PI-T1-FLC) [38] and PI type-2 FLC (PI-T2-FLC) [39]. One can see that the designed control system outperforms in a desirable performance. It needs to be noted that the mathematical dynamics of all units are considered to be unknown.

Scenario 2: For the second scenario, the parameters are fixed and a multiple load changes is considered. The other conditions are same as scenario 1. From Figure 8, the superiority of the designed controller is clearly seen. The trajectory of frequency for the suggested controller has a well convergence to zero. 

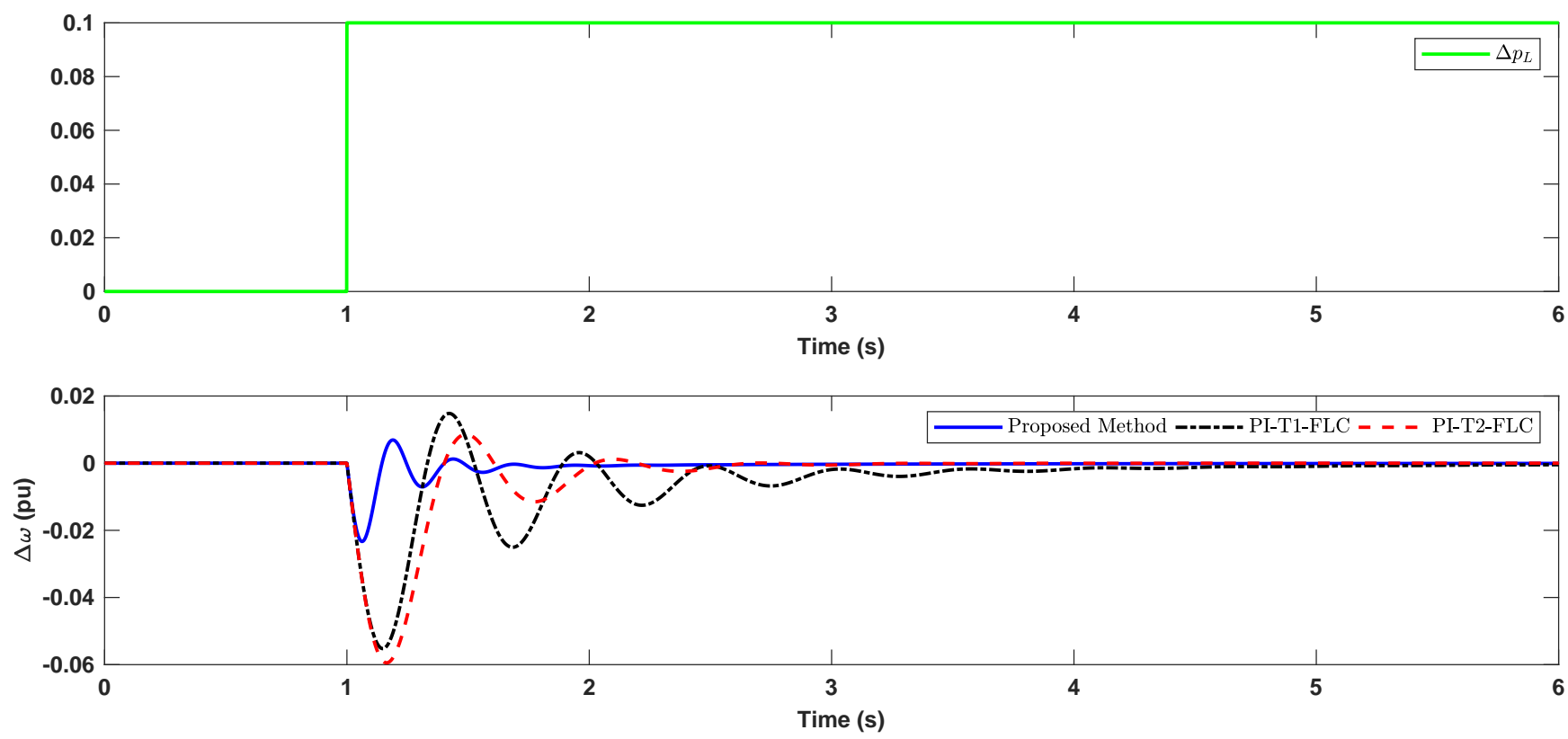

Figure 7. Example 1: Scenario 1: Frequency regulation performance in the presence load changes.
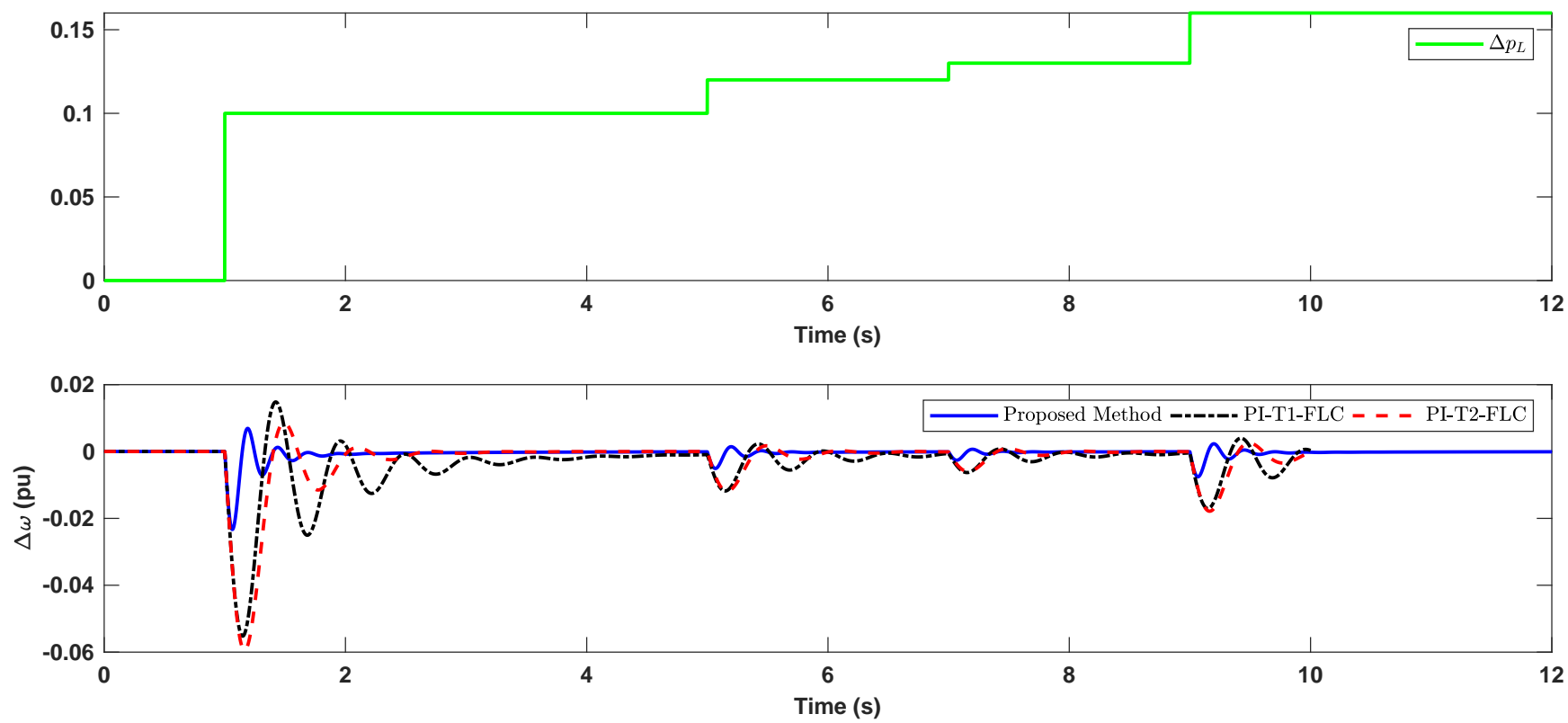

Figure 8. Example 1: Scenario 2: Frequency regulation performance in the presence of multiple load changes $\left(\Delta p_{L}\right)$.

Scenario 3: In the third scenario, in addition to the multiple load changes, the value of parameters of all units are considered to time-varying. All parameters are changed as $p=p(1+\sin (t))$. The regulation performance is given in Figure 9. The well proficiency of the suggested LFC can be significantly seen form Figure 9. 

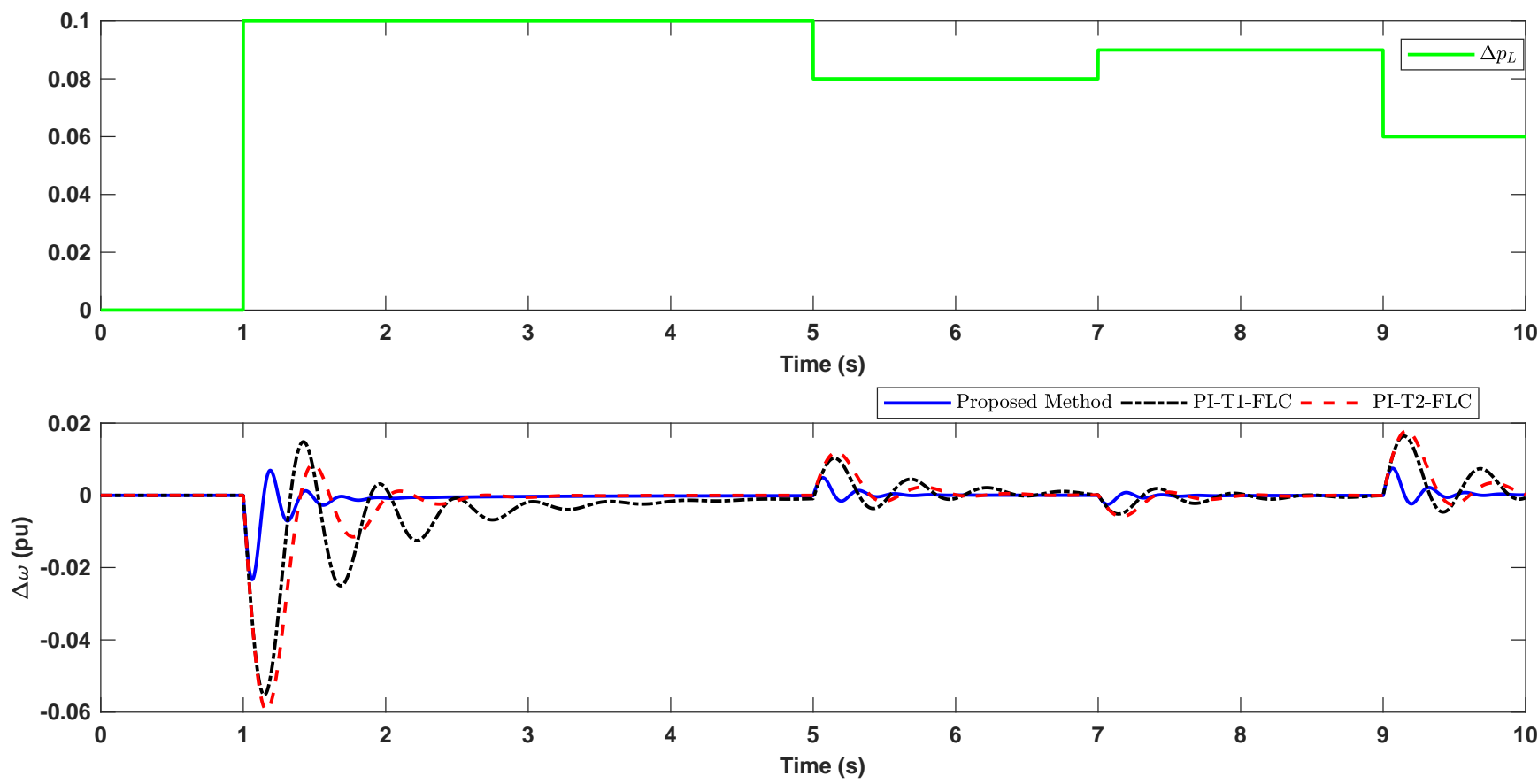

Figure 9. Example 1: Scenario 3: Frequency regulation in the presence of multiple load changes $\left(\Delta p_{L}\right)$, and all parameters are changed.

Scenario 4: In the fourth scenario, in addition to the multiple load changes, the solar radiation and mechanical power of wind turbine are also considered to be timevarying. The performance of the frequency in this scenario is depicted in Figure 10. One observes that the suggested method well resists versus the variation of solar radiation and perturbation of mechanical power of wind turbine and multiple load changes. It is seen that the trajectory of frequency corresponded to the suggested method has less deviation in contrast to the other controllers. The values of root mean square error (RMSE) for all scenarios are given in Table 3. It is perceived that the values for the suggested method in all scenarios is remarkably less than the other methods.
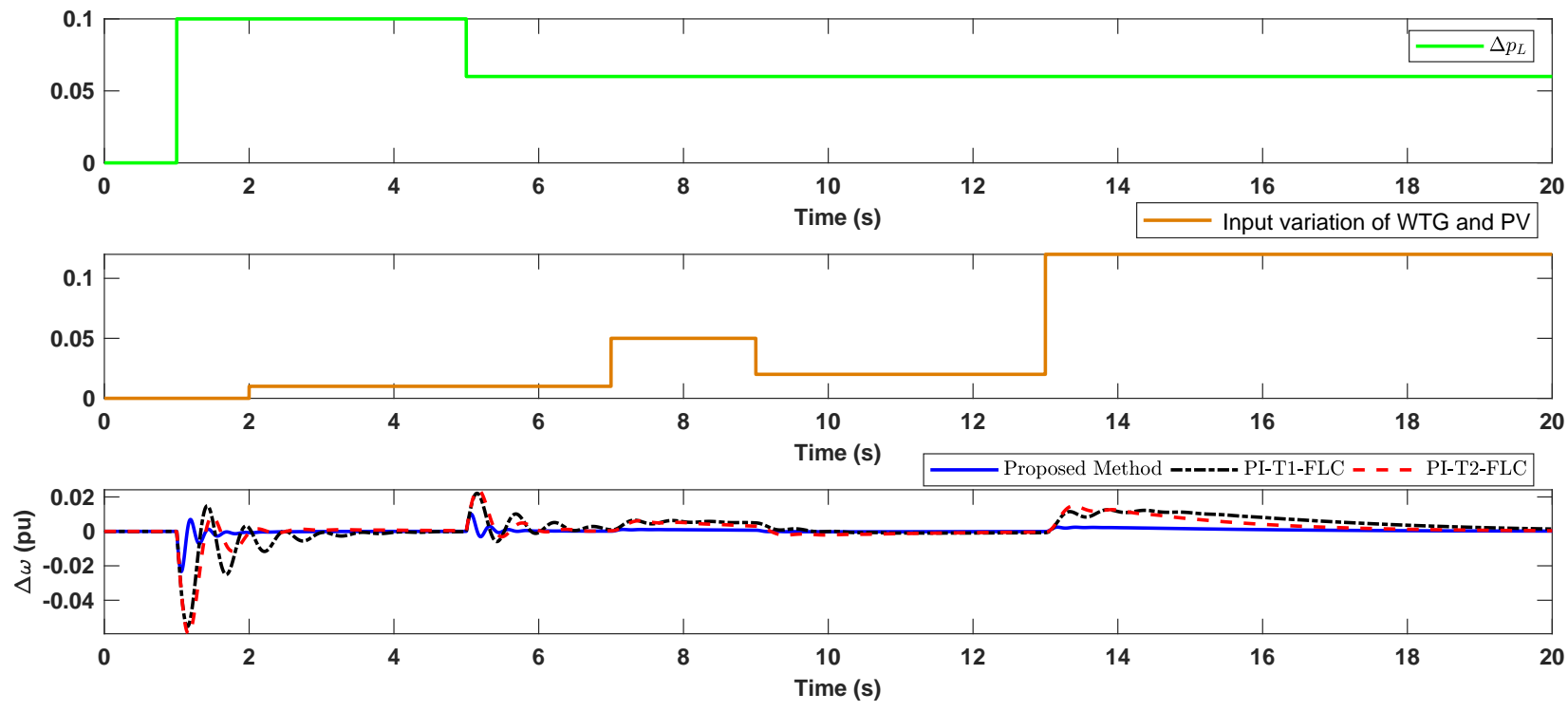

Figure 10. Example 1: Scenario 4: Frequency regulation performance in the presence of multiple load changes $\left(\Delta p_{L}\right)$ and time-varying solar radiation and mechanical power of wind turbine. 
Table 3. Regulation comparison by various controllers.

\begin{tabular}{cccc}
\hline \multirow{2}{*}{$\begin{array}{c}\text { RMSE } \\
\text { Scenario }\end{array}$} & Proposed Method & PI-T1-FLC & PI-T2-FLC \\
\cline { 2 - 4 } 1 & 0.0027 & 0.0105 & 0.0107 \\
2 & 0.0022 & 0.0084 & 0.0085 \\
3 & 0.0017 & 0.0064 & 0.0066 \\
4 & 0.0018 & 0.0078 & 0.0075 \\
\hline
\end{tabular}

Example 2. For the second example, the IEEE 39-bus test system is taken to account. The demand response for the first, second, and third area are considered as 98.77, 22.21, and 54.6 MW, respectively. The power capacity of all units are given in Table 4. The other conditions are same as [40]. The performance is examined in three scenarios.

Scenario 1: For the first scenario only load changes as shown in Figure 11 is considered and DR and storage systems and wind turbines are neglected. The frequency regulation performance for all three areas is given in Figure 12. It is observed that the trajectories of the suggested method in all three areas well converged to zero.

Table 4. Example 2: The value of powers in different areas.

\begin{tabular}{cccc}
\hline \multirow{2}{*}{ Area } & \multicolumn{3}{c}{ Power (MW) } \\
\cline { 2 - 4 } & Conventional & Wind & Load \\
\hline 1 & 134.57 & 61 & 329.25 \\
2 & 106.381 & 54 & 74.051 \\
3 & 163.9 & 72 & 182.01 \\
\hline
\end{tabular}

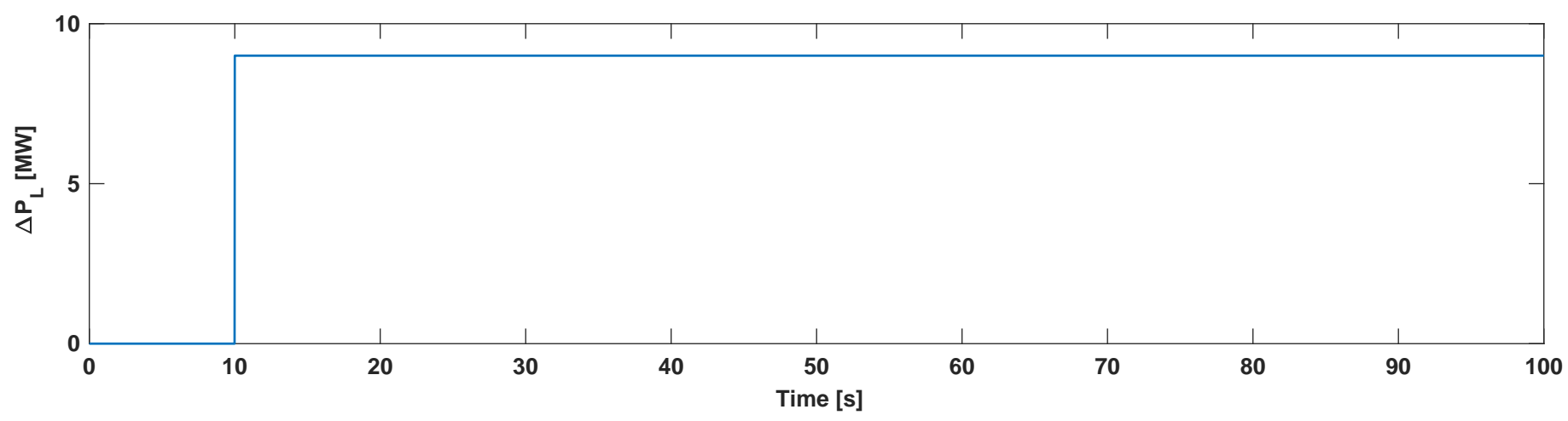

Figure 11. Example 2: The trajectory of load changes.

Scenario 2: For the second scenario, DR and storage systems are added to the previous condition and wind turbines are neglected. The frequency regulation performance in this condition is depicted in Figure 13. It is understood that the regulation performance corresponded to the suggested method is significantly better than PI-T1-FLC and PI-T2-FLC. 

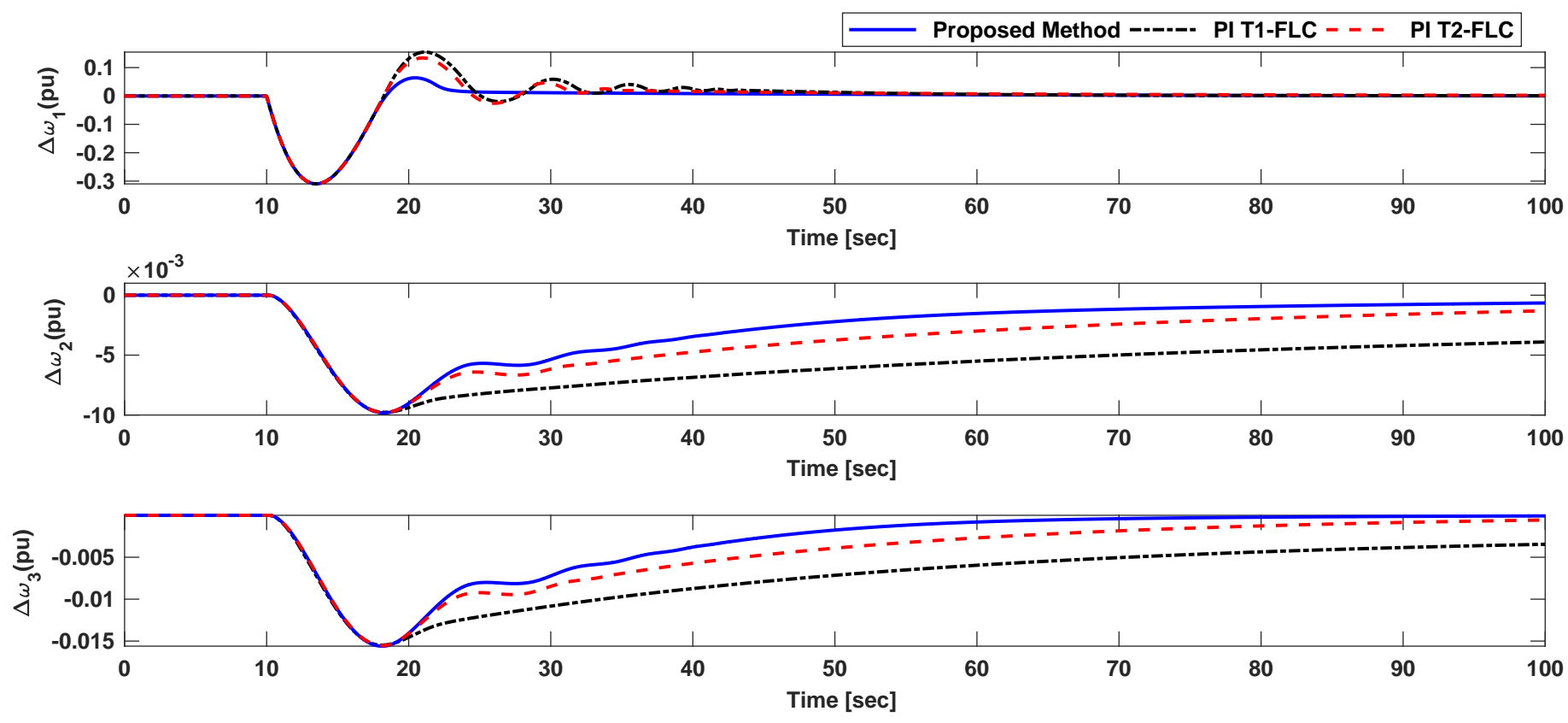

Figure 12. Example 2: Scenario 1: The trajectory of frequency regulation for all three areas.
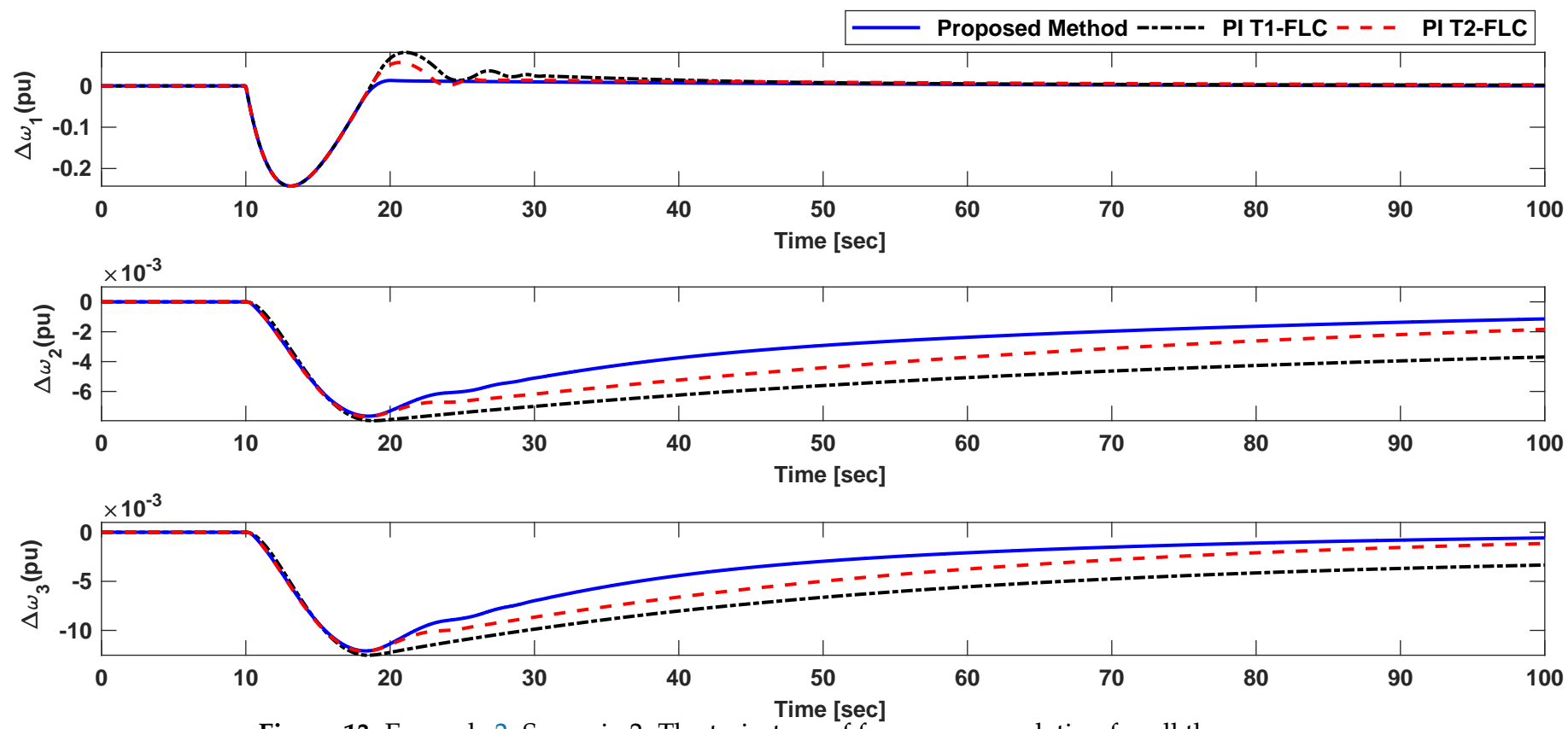

Figure 13. Example 2: Scenario 2: The trajectory of frequency regulation for all three areas.

Scenario 3: For the last scenario, the effect of time-varying wind speed is investigated. The wind speed trajectory is shown in Figure 14. The frequency regulation performance in this condition is given in Figure 15. We realize that the suggested controller resists well in the comparison of time-varying wind speed and also its regulation performance is better than conventional type- 1 and type-2 PI FLCs. The numerical comparison of RMSE for various control approaches and different scenarios are given in Table 5. It can be observed that the values of RMSE for the suggested controller is less than other fuzzy based controllers in all areas and all various conditions. 


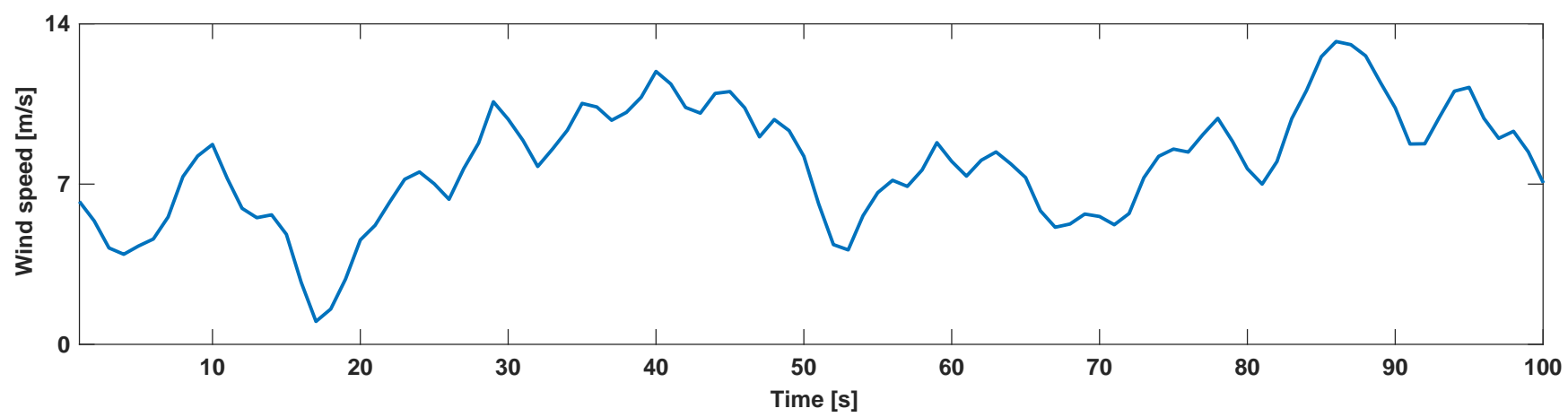

Figure 14. Example 2: Scenario 2: The trajectory of wind speed.
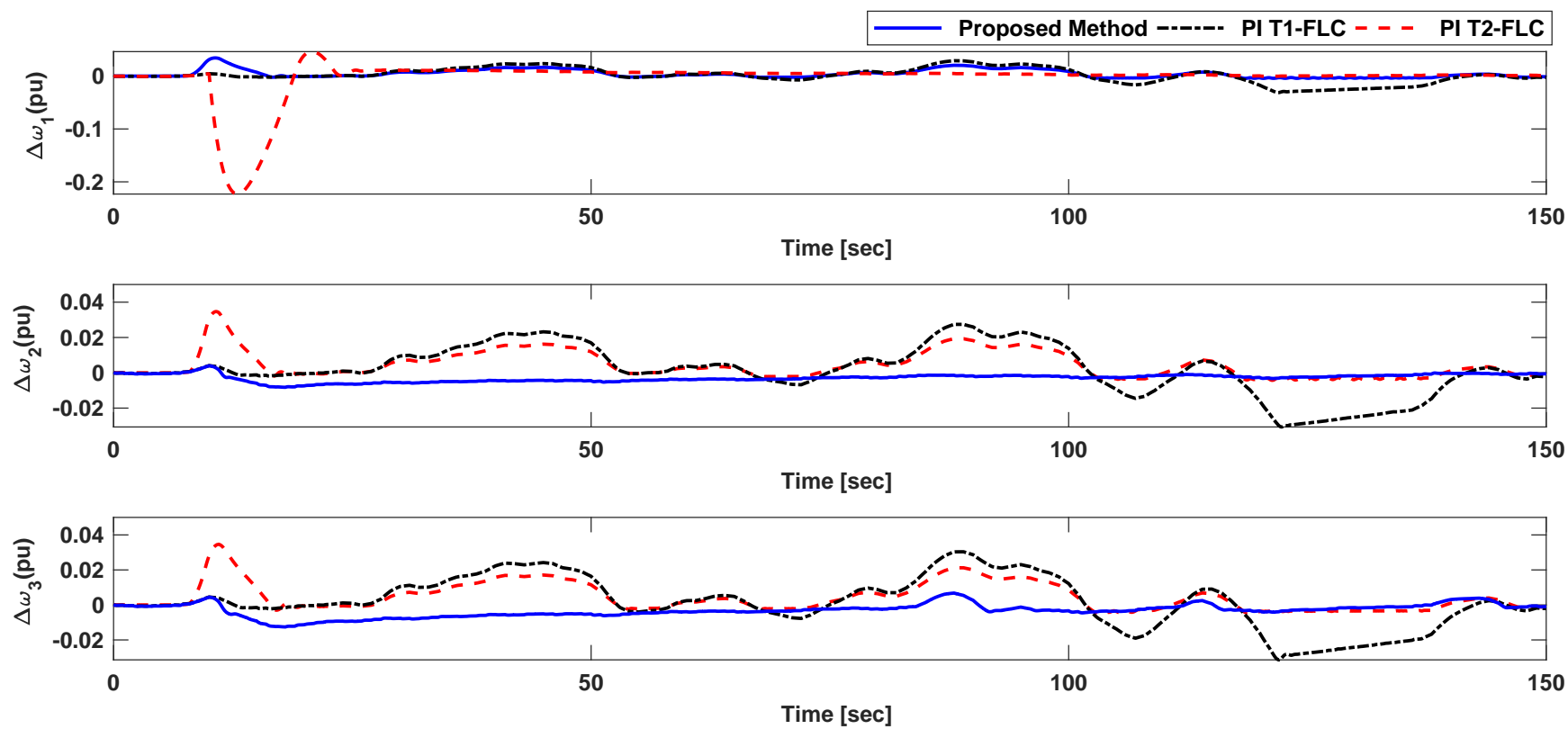

Figure 15. Example 2: Scenario 3: The trajectory of frequency regulation for all three areas.

Table 5. Example 2: Performance comparison for different control methods.

\begin{tabular}{ccccc}
\hline \multirow{2}{*}{ Scenario } & Controller & \multicolumn{3}{c}{ RMSE } \\
\cline { 3 - 5 } & & $\boldsymbol{\Delta} \boldsymbol{\omega}_{\mathbf{1}}$ & $\boldsymbol{\Delta} \boldsymbol{\omega}_{\mathbf{2}}$ & $\boldsymbol{\Delta} \boldsymbol{\omega}_{\mathbf{3}}$ \\
\hline \multirow{3}{*}{1} & Proposed FLC & 0.0641 & 0.0035 & 0.0050 \\
& PI-T1-FLC & 0.0709 & 0.0064 & 0.0083 \\
& PI-T2-FLC & 0.0688 & 0.0042 & 0.0056 \\
\hline \multirow{2}{*}{2} & Proposed FLC & 0.0510 & 0.0034 & 0.0046 \\
& PI-T1-FLC & 0.0534 & 0.0054 & 0.0070 \\
& PI-T2-FLC & 0.0519 & 0.0042 & 0.0055 \\
\hline \multirow{2}{*}{3} & Proposed FLC & 0.0457 & 0.0032 & 0.0043 \\
& PI-T1-FLC & 0.0474 & 0.0049 & 0.0063 \\
& PI-T2-FLC & 0.0463 & 0.0040 & 0.0052 \\
\hline
\end{tabular}

Remark 1. One of the main properties of the suggested LFC scheme is its good robustness against perturbations. As it shown in Figures 7-15, the trajectories of frequency deviation are approached to zero level at a finite time in the presence of hard perturbations such as multiple load changes, variation of solar energy, changes of wind speed, and dynamic parameter changes. 


\section{Conclusions}

In this study, a new adaptive FLC is designed for frequency regulation in multi-area power systems. The suggested controller is constructed by an error feedback controller, dynamic estimated model, and IT3-FLS. The gains of error feedback controller and the tuning rules of the dynamic model is extracted through the LMI stability analysis. The unknown dynamics of the units are estimated online by the suggested dynamic fractionalorder RBM and deep learning CD algorithm. The effectiveness of the schemed control approach is examined various conditions. For the first simulation example, an ac microgrid including loads, PVs, micro-turbines, WT, FESS, and BESS is considered and the performance is evaluated in four scenarios. For the first scenario, only small changes in the load is considered. In the second, multiple load changes is considered. For the third one, the issue of time-varying factors in all units is also added to the previous condition and finally in the fourth scenario the effect variation of irradiation and wind speed is examined. For the second simulation, the schemed controller is applied on the practical IEEE 39-bus system. Similar to the example one, the performance is evaluated in various conditions and effect of time-varying wind speed, multiple load changes, and DR and storage systems are investigated. In all cases the performance is compared with the some other popular controllers, such as PI-T1-FLC and PI-T2-FLC. One observes that suggested control system results in desirable frequency regulation performance in versus of various practical conditions and has clear superiority in contrast to the conventional FLCs.

Author Contributions: Conceptualization, A.M., O.C., A.A.A., B.F.F. and A.B.; Data curation, A.M., O.C., A.A.A., B.F.F. and A.B.; Formal analysis, A.M., O.C., A.A.A., B.F.F. and A.B.; Funding acquisition, A.A.A. and B.F.F.; Investigation, A.M., O.C., A.A.A., B.F.F. and A.B.; Methodology, A.M., O.C., A.A.A., B.F.F. and A.B.; Software, A.M., O.C., A.A.A., B.F.F. and A.B.; Supervision, A.M., O.C., A.A.A., B.F.F. and A.B.; Visualization, A.M., O.C., A.A.A., B.F.F. and A.B.; Writing-original draft, A.M., O.C., A.A.A., B.F.F. and A.B. All authors have read and agreed to the published version of the manuscript.

Funding: Taif University Researchers Supporting Project number (TURSP-2020/260), Taif University, Taif, Saudi Arabia.

Institutional Review Board Statement: Not applicable.

Informed Consent Statement: Not applicable.

Data Availability Statement: The study do not report any data.

Acknowledgments: The authors would like to express their sincere thanks to Rabia Safdar for his help in improving this paper.

Conflicts of Interest: The authors declare no conflict of interest.

\section{Appendix A. Stability Analysis}

The following Lemmas are applied to prove results of Theorem 1.

Lemma A1 ([41]). The following equations are equivalent for a real matrix $\Pi=\Pi^{T}$ :

(a) $\Pi=\left[\begin{array}{cc}\Pi_{11} & \Pi_{12} \\ * & \Pi_{22}\end{array}\right]>0$

(b) $\Pi_{11}>0$, and $\Pi_{22}-\Pi_{12}^{T} \Pi_{11}^{-1} \Pi_{12}>0$

(c) $\Pi_{22}>0$, and $\Pi_{11}-\Pi_{12}^{T} \Pi_{22}^{-1} \Pi_{12}>0$

Lemma A2 ([42]). Consider real matrices $U$ and $L$ and the following relation:

$$
U F(t) L+L^{T} F^{T}(t) U^{T}<0
$$


if $F^{T}(t) F(t) \leq I$, then there exists $a \vartheta$, such that

$$
\vartheta U U^{T}+\vartheta^{-1} L^{T} L<0
$$

Lemma A3 ([43]). The asymptotic stability of system $D_{t}^{q} x(t)=A x(t)$ is authenticated if there exist two symmetric matrices $\Pi_{j 1} \in \Re^{n \times n}, j=1,2$ with positive definite property and two skew-symmetric matrices $\Pi_{j 2} \in \Re^{n \times n}, j=1,2$, such that:

$$
\begin{aligned}
& \sum_{i=1}^{2} \sum_{j=1}^{2} \operatorname{Sym}\left\{\Theta_{i j} \otimes\left(A \Pi_{i j}\right)\right\}<0, \\
& {\left[\begin{array}{cc}
\Pi_{11} & \Pi_{12} \\
-\Pi_{12} & \Pi_{11}
\end{array}\right]>0, \quad\left[\begin{array}{cc}
\Pi_{21} & \Pi_{22} \\
-\Pi_{22} & \Pi_{21}
\end{array}\right]>0}
\end{aligned}
$$

where

$$
\begin{array}{ll}
\Theta_{11}=\left[\begin{array}{cc}
\sin \frac{\pi q}{2} & -\cos \frac{\pi q}{2} \\
\cos \frac{\pi q}{2} & \sin \frac{\pi q}{2}
\end{array}\right], & \Theta_{12}=\left[\begin{array}{cc}
\cos \frac{\pi q}{2} & \sin \frac{\pi q}{2} \\
-\sin \frac{\pi q}{2} & \cos \frac{\pi q}{2}
\end{array}\right] \\
\Theta_{21}=\left[\begin{array}{cc}
\sin \frac{\pi q}{2} & \cos \frac{\pi q}{2} \\
-\cos \frac{\pi q}{2} & \sin \frac{\pi q}{2}
\end{array}\right], & \Theta_{22}=\left[\begin{array}{cc}
-\cos \frac{\pi q}{2} & \sin \frac{\pi q}{2} \\
-\sin \frac{\pi q}{2} & -\cos \frac{\pi q}{2}
\end{array}\right]
\end{array}
$$

$\operatorname{Sym}\{X\}=X+X^{T}$ and $\otimes$ is Kronecker product.

From the universal approximation feature of neural networks and by considering the optimal MLP the dynamics of $\Delta \omega_{k}$ can be written as:

$$
D_{t}^{q} \Delta \omega_{k}=\hat{f}_{k}^{*}\left(x_{k} \mid \theta_{k m}^{*}, \xi_{k}, \varepsilon_{k}, \eta_{k}\right)+E_{k}+u_{k}
$$

where, $\theta_{k m}^{*}$ is the vector of optimal parameters and $E_{k}$ is the approximation error. From (8) and (A2), the dynamic of estimation error $\Delta \tilde{\omega}_{k}$ is obtained as:

$$
D_{t}^{q} \Delta \tilde{\omega}_{k}=\hat{f}_{k}^{*}\left(x_{k} \mid \theta_{m}^{*}, \xi_{k}, \varepsilon_{k}, \eta_{k}\right)-\hat{f}_{k}\left(x_{k} \mid \theta_{m}, \xi_{k}, \varepsilon_{k}, \eta_{k}\right)-\chi_{k} \Delta \tilde{\omega}_{k}+E_{k}
$$

where, $\Delta \tilde{\omega}_{k}=\Delta \omega_{k}-\Delta \hat{\omega}_{k}$. Consider the flowing definitions:

$$
\begin{aligned}
& \tilde{\theta}_{m}=\theta_{m}^{*}-\theta_{m} \\
& \hat{f}_{k}^{*}\left(x_{k} \mid \theta_{m}^{*}, \xi_{k}, \varepsilon_{k}, \eta_{k}\right)-\hat{f}_{k}\left(x_{k} \mid \theta_{m}, \xi_{k}, \varepsilon_{k}, \eta_{k}\right)=\tilde{\theta}_{m}^{T} H_{k 1}
\end{aligned}
$$

From (A4), $D_{t}^{q} \Delta \tilde{\omega}_{k}$ in (A3) is rewritten as:

$$
D_{t}^{q} \Delta \tilde{\omega}_{k}=\tilde{\theta}_{m}^{T} H_{k 1}-\chi_{k} \Delta \tilde{\omega}_{k}+E_{k}
$$

The approximation error $E_{k}$ can be written as:

$$
E_{k}=D_{k} \phi_{k}(t) \Delta \hat{\omega}_{k}
$$

where, $\bar{E}_{k}$ is the upper bound of $E_{k}$ and $0 \leq \phi_{k}(t) \leq 1$. From (A6), the Equation (A5) is rewritten as:

$$
D_{t}^{q} \Delta \tilde{\omega}_{k}=\tilde{\theta}_{m}^{T} H_{k 1}-\chi_{k} \Delta \tilde{\omega}_{k}+\bar{E}_{k} \phi_{k}(t) \Delta \hat{\omega}_{k}
$$

By applying the control signal (40), the dynamic of $\Delta \hat{\omega}_{k}$ becomes:

$$
D_{t}^{q} \Delta \hat{\omega}_{k}=-\lambda_{k} \Delta \omega_{k}+\chi_{k} \Delta \tilde{\omega}_{k}-u_{c k}\left(x_{c k} \mid \theta_{c k}\right)
$$

By substituting $u_{c k}\left(x_{c k} \mid \theta_{c k}\right)$ from (41), one has:

$$
D_{t}^{q} \Delta \hat{\omega}_{k}=-\lambda_{k} \Delta \omega_{k}+\chi_{k} \Delta \tilde{\omega}_{k}-\varphi_{c k}^{T} \theta_{c k} \Delta \hat{\omega}_{k}
$$


In the vector form, one has:

$$
D_{t}^{q}\left[\begin{array}{c}
\Delta \hat{\omega}_{k} \\
\Delta \tilde{\omega}_{k} \\
\tilde{\theta}_{m}
\end{array}\right]=\left[\begin{array}{ccc}
-\lambda_{k} & \chi_{k} & \underline{0}_{1 \times n} \\
0 & -\chi_{k} & H_{k 1}^{T} \\
\underline{0}_{n \times 1} & -\gamma H_{k 1} & \underline{0}_{n \times n}
\end{array}\right]\left[\begin{array}{c}
\Delta \hat{\omega}_{k} \\
\Delta \tilde{\omega}_{k} \\
\tilde{\theta}_{m}
\end{array}\right]+\left[\begin{array}{c}
-u_{c k}\left(x_{c k} \mid \theta_{c}\right) \\
E_{k} \\
0
\end{array}\right]
$$

where, $n$ is number of elements in $H_{k 1}$.

Considering (Lemma A3) and by choosing $P_{12}=P_{22}=0$ and

$$
P_{11}=P_{21}=\left[\begin{array}{ccc}
Q_{1} & 0 & \underline{0}_{1 \times n} \\
0 & Q_{2} & \underline{0}_{1 \times n} \\
\underline{0}_{n \times 1} & \underline{0}_{n \times 1} & Q_{3}
\end{array}\right]
$$

It is derived that system (A10) is asymptotically stable if:

$$
\sum_{i=1}^{2} \operatorname{Sym}\left\{\Theta_{i 1} \otimes\left[\begin{array}{ccc}
-\lambda_{k}-\varphi_{c k}^{T} \theta_{c k} & \chi_{k} & \underline{0}_{1 \times n} \\
\bar{E}_{k} \phi_{k}(t) & -\chi_{k} & H_{k 1}^{T} \\
\underline{0}_{n \times 1} & -\gamma H_{k 1} & \underline{0}_{n \times n}
\end{array}\right]\left[\begin{array}{ccc}
Q_{1} & 0 & \underline{0}_{1 \times n} \\
0 & Q_{2} & \underline{0}_{1 \times n} \\
\underline{0}_{n \times 1} & \underline{0}_{n \times 1} & Q_{3}
\end{array}\right]\right\}<0
$$

The inequality (A12) can be rewritten as:

$$
\begin{aligned}
& \sum_{i=1}^{2} \operatorname{Sym}\left\{\Theta_{i 1} \otimes\left[\begin{array}{ccc}
-\lambda_{k} Q_{1}-\varphi_{c k}^{T} \theta_{c k} Q_{1} & \chi_{k} Q_{2} & \underline{0}_{1 \times n} \\
0 & -\chi_{k} Q_{2} & H_{k 1}^{T} Q_{3} \\
\underline{0}_{n \times 1} & -\gamma H_{k 1} Q_{2} & \underline{n}_{n \times n}
\end{array}\right]\right\} \\
& +\sum_{i=1}^{2} \operatorname{Sym}\left\{\Theta_{i 1} \otimes\left[\begin{array}{ccc}
0 & 0 & \underline{0}_{1 \times n} \\
\bar{E}_{k} \phi_{k}(t) Q_{1} & 0 & \underline{0}_{1 \times n} \\
\underline{0}_{n \times 1} & \underline{0}_{n \times 1} & \underline{0}_{n \times n}
\end{array}\right]\right\}<0
\end{aligned}
$$

From (A13), one has:

$$
\begin{aligned}
& \sum_{i=1}^{2} \operatorname{Sym}\left\{\Theta_{i 1} \otimes\left[\begin{array}{ccc}
0 & 0 & \underline{0}_{1 \times n} \\
\bar{E}_{k} \phi_{k}(t) Q_{1} & 0 & \underline{0}_{1 \times n} \\
\underline{0}_{n \times 1} & \underline{0}_{n \times 1} & \underline{0}_{n \times n}
\end{array}\right]\right\}= \\
& \sum_{i=1}^{2} \operatorname{Sym}\left\{\Theta_{i 1} \otimes\left(\left[\begin{array}{cccc}
0 & 0 & \underline{0}_{1 \times n} \\
\bar{E}_{k} & 0 & \underline{0}_{1 \times n} \\
\underline{0}_{n \times 1} & \underline{0}_{n \times 1} & \underline{0}_{n \times n}
\end{array}\right]\left[\begin{array}{ccc}
\phi_{k}(t) & 0 & \underline{0}_{1 \times n} \\
0 & \phi_{k}(t) & \underline{0}_{1 \times n} \\
\underline{0}_{n \times 1} & \underline{0}_{n \times 1} & \underline{0}_{n \times n}
\end{array}\right]\left[\begin{array}{ccc}
Q_{1} & 0 & \underline{0}_{1 \times n} \\
0 & Q_{1} & \underline{0}_{1 \times n} \\
\underline{0}_{n \times 1} & \underline{0}_{n \times 1} & \underline{0}_{n \times n}
\end{array}\right]\right)\right\}
\end{aligned}
$$

From Equation (A14), the second term is rewritten as:

$$
\begin{aligned}
& \sum_{i=1}^{2} \operatorname{Sym}\left\{\Theta_{i 1} \otimes\left(\left[\begin{array}{ccc}
0 & 0 & \underline{0}_{1 \times n} \\
\bar{E}_{k} & 0 & \underline{0}_{1 \times n} \\
\underline{0}_{n \times 1} & \underline{0}_{n \times 1} & \underline{0}_{n \times n}
\end{array}\right]\left[\begin{array}{ccc}
\phi_{k}(t) & 0 & \underline{0}_{1 \times n} \\
0 & \phi_{k}(t) & \underline{0}_{1 \times n} \\
\underline{0}_{n \times 1} & \underline{0}_{n \times 1} & \underline{0}_{n \times n}
\end{array}\right]\left[\begin{array}{ccc}
Q_{1} & 0 & \underline{0}_{1 \times n} \\
0 & Q_{1} & \underline{0}_{1 \times n} \\
\underline{0}_{n \times 1} & \underline{0}_{n \times 1} & \underline{0}_{n \times n}
\end{array}\right]\right)\right\}= \\
& \sum_{i=1}^{2} \operatorname{Sym}\left\{\left(\Theta_{i 1} \otimes\left[\begin{array}{ccc}
0 & 0 & \underline{0}_{1 \times n} \\
\bar{E}_{k} & 0 & \underline{0}_{1 \times n} \\
\underline{0}_{n \times 1} & \underline{0}_{n \times 1} & \underline{0}_{n \times n}
\end{array}\right]\right)\right. \text {. } \\
& \left(I_{(n+2)} \otimes\left[\begin{array}{ccc}
\phi_{k}(t) & 0 & \underline{0}_{1 \times n} \\
0 & \phi_{k}(t) & \underline{0}_{1 \times n} \\
\underline{0}_{n \times 1} & \underline{0}_{n \times 1} & \underline{0}_{n \times n}
\end{array}\right]\right) \text {. } \\
& \left.\left(I_{(n+2)} \otimes\left[\begin{array}{ccc}
Q_{1} & 0 & \underline{0}_{1 \times n} \\
0 & Q_{1} & \underline{0}_{1 \times n} \\
\underline{0}_{n \times 1} & \underline{0}_{n \times 1} & \underline{0}_{n \times n}
\end{array}\right]\right)\right\}
\end{aligned}
$$


From (A15), it can be written:

$$
\begin{aligned}
& \left(I_{(n+2)} \otimes\left[\begin{array}{ccc}
\phi_{k}(t) & 0 & \underline{0}_{1 \times n} \\
0 & \phi_{k}(t) & \underline{0}_{1 \times n} \\
\underline{0}_{n \times 1} & \underline{0}_{n \times 1} & \underline{0}_{n \times n}
\end{array}\right]\right)\left(I_{(n+2)} \otimes\left[\begin{array}{ccc}
\phi_{k}(t) & 0 & \underline{0}_{1 \times n} \\
0 & \phi_{k}(t) & \underline{0}_{1 \times n} \\
\underline{0}_{n \times 1} & \underline{0}_{n \times 1} & \underline{0}_{n \times n}
\end{array}\right]\right)^{T}= \\
& \left(I_{(n+2)} \otimes\left[\begin{array}{ccc}
\phi_{k}(t) \phi_{k}^{T}(t) & 0 & \underline{0}_{1 \times n} \\
0 & \phi_{k}(t) \phi_{k}^{T}(t) & \underline{0}_{1 \times n} \\
\underline{0}_{n \times 1} & \underline{0}_{n \times 1} & \underline{0}_{n \times n}
\end{array}\right]\right) \leq I_{(n+2)}
\end{aligned}
$$

Then form (A16) and Lemma A2, the equation (A14) is rewritten as:

$$
\begin{aligned}
& \sum_{i=1}^{2} \operatorname{Sym}\left\{\Theta_{i 1} \otimes\left[\begin{array}{ccc}
0 & 0 & \underline{0}_{1 \times n} \\
\bar{E}_{k} \phi_{k}(t) Q_{1} & 0 & \underline{0}_{1 \times n} \\
\underline{0}_{n \times 1} & \underline{\underline{n}}_{n \times 1} & \underline{0}_{n \times n}
\end{array}\right]\right\} \\
& \sum_{i=1}^{2} \vartheta_{i}\left(\Theta_{i 1} \otimes\left[\begin{array}{ccc}
0 & 0 & \underline{0}_{1 \times n} \\
\bar{E}_{k} & 0 & \underline{0}_{1 \times n} \\
\underline{0}_{n \times 1} & \underline{0}_{n \times 1} & \underline{0}_{n \times n}
\end{array}\right]\right) \cdot\left(\Theta_{i 1} \otimes\left[\begin{array}{ccc}
0 & 0 & \underline{0}_{1 \times n} \\
\bar{E}_{k} & 0 & \underline{0}_{1 \times n} \\
\underline{0}_{n \times 1} & \underline{0}_{n \times 1} & \underline{0}_{n \times n}
\end{array}\right]\right) \\
& +\vartheta_{i}^{-1}\left(I_{(n+2)} \otimes\left[\begin{array}{ccc}
Q_{1} & 0 & \underline{0}_{1 \times n} \\
0 & Q_{1} & \underline{0}_{1 \times n} \\
\underline{0}_{n \times 1} & \underline{0}_{n \times 1} & \underline{0}_{n \times n}
\end{array}\right]\right) \cdot\left(I_{(n+2)} \otimes\left[\begin{array}{ccc}
Q_{1} & 0 & \underline{0}_{1 \times n} \\
0 & Q_{1} & \underline{0}_{1 \times n} \\
\underline{0}_{n \times 1} & \underline{0}_{n \times 1} & \underline{0}_{n \times n}
\end{array}\right]\right)
\end{aligned}
$$

From (A17), the inequality (A13) becomes:

$$
\begin{aligned}
& \sum_{i=1}^{2} \operatorname{Sym}\left\{\Theta_{i 1} \otimes\left[\begin{array}{ccc}
-\lambda_{k} Q_{1}-\varphi_{c k}^{T} \theta_{c k} Q_{1} & \chi_{k} Q_{2} & \underline{0}_{1 \times n} \\
0 & -\chi_{k} Q_{2} & H_{k 1}^{T} Q_{3} \\
\underline{0}_{n \times 1} & -\gamma H_{k 1} Q_{2} & \underline{0}_{n \times n}
\end{array}\right]\right\}+ \\
& \sum_{i=1}^{2} \vartheta_{i}\left(\Theta_{i 1} \otimes\left[\begin{array}{ccc}
0 & 0 & \underline{0}_{1 \times n} \\
\bar{E}_{k} & 0 & \underline{0}_{1 \times n} \\
\underline{n}_{n \times 1} & \underline{0}_{n \times 1} & \underline{0}_{n \times n}
\end{array}\right]\right) \cdot\left(\Theta_{i 1} \otimes\left[\begin{array}{ccc}
0 & 0 & \underline{0}_{1 \times n} \\
\bar{E}_{k} & 0 & \underline{0}_{1 \times n} \\
\underline{0}_{n \times 1} & \underline{0}_{n \times 1} & \underline{0}_{n \times n}
\end{array}\right]\right) \\
& +\vartheta_{i}^{-1}\left(I_{(n+2)} \otimes\left[\begin{array}{ccc}
Q_{1} & 0 & \underline{0}_{1 \times n} \\
0 & Q_{1} & \underline{0}_{1 \times n} \\
\underline{0}_{n \times 1} & \underline{0}_{n \times 1} & \underline{0}_{n \times n}
\end{array}\right]\right) \cdot\left(I_{(n+2)} \otimes\left[\begin{array}{ccc}
Q_{1} & 0 & \underline{0}_{1 \times n} \\
0 & Q_{1} & \underline{0}_{1 \times n} \\
\underline{0}_{n \times 1} & \underline{0}_{n \times 1} & \underline{0}_{n \times n}
\end{array}\right]\right)<0
\end{aligned}
$$

By considering $X_{k 1}=\lambda_{k} Q_{1}, X_{k 2}=\chi_{k} Q_{2}$ and $\Theta_{i 1}^{T} \Theta_{i 1}=I_{2}$, the inequality (A18), becomes:

$$
\begin{aligned}
& \sum_{i=1}^{2} \operatorname{Sym}\left\{\Theta_{i 1} \otimes\left[\begin{array}{ccc}
-X_{k 1}-\varphi_{c k}^{T} \theta_{c k} Q_{1} & X_{k 2} & \underline{0}_{1 \times n} \\
0 & -X_{k 2} & H_{k 1}^{T} Q_{3} \\
\underline{0}_{n \times 1} & -\gamma H_{k 1} Q_{2} & \underline{0}_{n \times n}
\end{array}\right]\right\}+ \\
& \sum_{i=1}^{2} \vartheta_{i}\left(I_{2} \otimes\left[\begin{array}{ccc}
0 & 0 & \underline{0}_{1 \times n} \\
\bar{E}_{k} \bar{E}_{k}^{T} & 0 & \underline{0}_{1 \times n} \\
\underline{0}_{n \times 1} & \underline{0}_{n \times 1} & \underline{0}_{n \times n}
\end{array}\right]\right) \\
& +\vartheta_{i}^{-1}\left(I_{(n+2)} \otimes\left[\begin{array}{ccc}
Q_{1} & 0 & \underline{0}_{1 \times n} \\
0 & Q_{1} & \underline{0}_{1 \times n} \\
\underline{0}_{n \times 1} & \underline{0}_{n \times 1} & \underline{0}_{n \times n}
\end{array}\right]\right) \cdot\left(I_{(n+2)} \otimes\left[\begin{array}{ccc}
Q_{1} & 0 & \underline{0}_{1 \times n} \\
0 & Q_{1} & \underline{0}_{1 \times n} \\
\underline{0}_{n \times 1} & \underline{0}_{n \times 1} & \underline{0}_{n \times n}
\end{array}\right]\right)<0
\end{aligned}
$$

From (A19), one can has:

$$
\begin{aligned}
& \sum_{i=1}^{2} \vartheta_{i}^{-1}\left(I_{(n+2)} \otimes\left[\begin{array}{ccc}
Q_{1} & 0 & \underline{0}_{1 \times n} \\
0 & Q_{1} & \underline{0}_{1 \times n} \\
\underline{0}_{n \times 1} & \underline{0}_{n \times 1} & \underline{0}_{n \times n}
\end{array}\right]\right) \cdot\left(I_{(n+2)} \otimes\left[\begin{array}{ccc}
Q_{1} & 0 & \underline{0}_{1 \times n} \\
0 & Q_{1} & \underline{0}_{1 \times n} \\
\underline{0}_{n \times 1} & \underline{0}_{n \times 1} & \underline{0}_{n \times n}
\end{array}\right]\right)= \\
& \left(I_{(n+2)} \otimes\left[\begin{array}{ccc}
Q_{1} & 0 & \underline{0}_{1 \times n} \\
0 & Q_{1} & \underline{0}_{1 \times n} \\
\underline{0}_{n \times 1} & \underline{0}_{n \times 1} & \underline{0}_{n \times n}
\end{array}\right]\right) \vartheta_{1}^{-1}\left(I_{(n+2)} \otimes\left[\begin{array}{ccc}
Q_{1} & 0 & \underline{0}_{1 \times n} \\
0 & Q_{1} & \underline{0}_{1 \times n} \\
\underline{0}_{n \times 1} & \underline{0}_{n \times 1} & \underline{0}_{n \times n}
\end{array}\right]\right)+
\end{aligned}
$$




$$
\begin{aligned}
& \left(I_{(n+2)} \otimes\left[\begin{array}{ccc}
Q_{1} & 0 & \underline{0}_{1 \times n} \\
0 & Q_{1} & \underline{0}_{1 \times n} \\
\underline{0}_{n \times 1} & \underline{0}_{n \times 1} & \underline{0}_{n \times n}
\end{array}\right]\right)^{T} \vartheta_{2}^{-1}\left(I_{(n+2)} \otimes\left[\begin{array}{ccc}
Q_{1} & 0 & \underline{0}_{1 \times n} \\
0 & Q_{1} & \underline{0}_{1 \times n} \\
\underline{n}_{n \times 1} & \underline{0}_{n \times 1} & \underline{0}_{n \times n}
\end{array}\right]\right)= \\
& {\left[\left(I_{(n+2)} \otimes\left[\begin{array}{ccc}
Q_{1} & 0 & \underline{0}_{1 \times n} \\
0 & Q_{1} & \underline{0}_{1 \times n} \\
\underline{0}_{n \times 1} & \underline{0}_{n \times 1} & \underline{0}_{n \times n}
\end{array}\right]\right)^{T}\left(I_{(n+2)} \otimes\left[\begin{array}{ccc}
Q_{1} & 0 & \underline{0}_{1 \times n} \\
0 & Q_{1} & \underline{0}_{1 \times n} \\
\underline{0}_{n \times 1} & \underline{0}_{n \times 1} & \underline{0}_{n \times n}
\end{array}\right]\right)\right]^{T} .} \\
& \left(\left[\begin{array}{cc}
\vartheta_{1} & 0 \\
0 & \vartheta_{2}
\end{array}\right] \otimes I_{2}\right)^{-1} \cdot \\
& {\left[\left(I_{(n+2)} \otimes\left[\begin{array}{ccc}
Q_{1} & 0 & \underline{0}_{1 \times n} \\
0 & Q_{1} & \underline{0}_{1 \times n} \\
\underline{0}_{n \times 1} & \underline{0}_{n \times 1} & \underline{0}_{n \times n}
\end{array}\right]\right)^{T}\left(I_{(n+2)} \otimes\left[\begin{array}{ccc}
Q_{1} & 0 & \underline{0}_{1 \times n} \\
0 & Q_{1} & \underline{0}_{1 \times n} \\
\underline{0}_{n \times 1} & \underline{0}_{n \times 1} & \underline{0}_{n \times n}
\end{array}\right]\right)\right]}
\end{aligned}
$$

From Equations (A19) and (A20) and Lemma A1, the inequality (36) is obtained. This completes the proof.

\section{References}

1. Mihet-Popa, L.; Saponara, S. Power Converters, Electric Drives and Energy Storage Systems for Electrified Transportation and Smart Grid Applications. Energies 2021, 14, 4142. [CrossRef]

2. Subramanian, S.; Sankaralingam, C.; Elavarasan, R.M.; Vijayaraghavan, R.R.; Raju, K.; Mihet-Popa, L. An Evaluation on Wind Energy Potential Using Multi-Objective Optimization Based Non-Dominated Sorting Genetic Algorithm III. Sustainability 2021, 13, 410. [CrossRef]

3. Armghan, A.; Azeem, M.K.; Armghan, H.; Yang, M.; Alenezi, F.; Hassan, M. Dynamical Operation Based Robust Nonlinear Control of DC Microgrid Considering Renewable Energy Integration. Energies 2021, 14, 3988. [CrossRef]

4. Marti-Puig, P.; Blanco-M, A.; Cárdenas, J.J.; Cusidó, J.; Solé-Casals, J. Feature selection algorithms for wind turbine failure prediction. Energies 2019, 12, 453. [CrossRef]

5. Armghan, A.; Hassan, M.; Armghan, H.; Yang, M.; Alenezi, F.; Azeem, M.K.; Ali, N. Barrier Function Based Adaptive Sliding Mode Controller for a Hybrid AC/DC Microgrid Involving Multiple Renewables. Appl. Sci. 2021, 11, 8672. [CrossRef]

6. Shabani, H.; Vahidi, B.; Ebrahimpour, M. A robust PID controller based on imperialist competitive algorithm for load-frequency control of power systems. ISA Trans. 2013, 52, 88-95. [CrossRef]

7. Farahani, M.; Ganjefar, S.; Alizadeh, M. PID controller adjustment using chaotic optimisation algorithm for multi-area load frequency control. IET Control Theory Appl. 2012, 6, 1984-1992. [CrossRef]

8. Wies, R.W.; Chukkapalli, E.; Mueller-Stoffels, M. Improved frequency regulation in mini-grids with high wind contribution using online genetic algorithm for PID tuning. In Proceedings of the 2014 IEEE PES General Meeting I Conference \& Exposition, IEEE, New York, NY, USA, 27-31 July 2014; pp. 1-5.

9. Sahoo, B.; Panda, S. Improved grey wolf optimization technique for fuzzy aided PID controller design for power system frequency control. Sustain. Energy Grids Netw. 2018, 16, 278-299. [CrossRef]

10. Abedinia, O.; Naderi, M.S.; Ghasemi, A. Robust LFC in deregulated environment: Fuzzy PID using HBMO. In Proceedings of the 2011 10th International Conference on Environment and Electrical Engineering, IEEE, New York, NY, USA, 8-11 May 2011; pp. 1-4.

11. Ali, E.; Abd-Elazim, S. BFOA based design of PID controller for two area load frequency control with nonlinearities. Int. J. Electr. Power Energy Syst. 2013, 51, 224-231. [CrossRef]

12. Kouba, N.E.Y.; Menaa, M.; Hasni, M.; Boudour, M. LFC enhancement concerning large wind power integration using new optimised PID controller and RFBs. IET Gener. Transm. Distrib. 2016, 10, 4065-4077. [CrossRef]

13. Kontogiannis, D.; Bargiotas, D.; Daskalopulu, A. Fuzzy control system for smart energy management in residential buildings based on environmental data. Energies 2021, 14, 752. [CrossRef]

14. Kontogiannis, D.; Bargiotas, D.; Daskalopulu, A. Minutely active power forecasting models using neural networks. Sustainability 2020, 12, 3177. [CrossRef]

15. Cusidó, J.; López, A.; Beretta, M. Fault-Tolerant Control of a Wind Turbine Generator Based on Fuzzy Logic and Using Ensemble Learning. Energies 2021, 14, 5167. [CrossRef]

16. Yesil, E. Interval type-2 fuzzy PID load frequency controller using Big Bang-Big Crunch optimization. Appl. Soft Comput. 2014, 15, 100-112. [CrossRef]

17. Khooban, M.H.; Niknam, T. A new intelligent online fuzzy tuning approach for multi-area load frequency control: Self Adaptive Modified Bat Algorithm. Int. J. Electr. Power Energy Syst. 2015, 71, 254-261. [CrossRef]

18. Sahu, B.K.; Pati, S.; Mohanty, P.K.; Panda, S. Teaching-learning based optimization algorithm based fuzzy-PID controller for automatic generation control of multi-area power system. Appl. Soft Comput. 2015, 27, 240-249. [CrossRef] 
19. Gheisarnejad, M. An effective hybrid harmony search and cuckoo optimization algorithm based fuzzy PID controller for load frequency control. Appl. Soft Comput. 2018, 65, 121-138. [CrossRef]

20. Kouba, N.E.Y.; Menaa, M.; Hasni, M.; Boudour, M. Application of multi-verse optimiser-based fuzzy-PID controller to improve power system frequency regulation in presence of HVDC link. Int. J. Intell. Eng. Inform. 2018, 6, 182-203. [CrossRef]

21. Chintu, J.M.R.; Sahu, R.K. Differential Evolution Optimized Fuzzy PID Controller for Automatic Generation Control of Interconnected Power System. In Computational Intelligence in Pattern Recognition; Springer: Berlin/Heidelberg, Germany, 2020; pp. 123-132.

22. Arya, Y. AGC of two-area electric power systems using optimized fuzzy PID with filter plus double integral controller. J. Frankl. Inst. 2018, 355, 4583-4617. [CrossRef]

23. Jena, T.; Debnath, M.K.; Sanyal, S.K. Optimal fuzzy-PID controller with derivative filter for load frequency control including UPFC and SMES. Int. J. Electr. Comput. Eng. 2019, 9, 2813. [CrossRef]

24. Debnath, M.K.; Jena, T.; Sanyal, S.K. Frequency control analysis with PID-fuzzy-PID hybrid controller tuned by modified GWO technique. Int. Trans. Electr. Energy Syst. 2019, 29, e12074. [CrossRef]

25. Khamari, D.; Sahu, R.K.; Panda, S. A Modified Moth Swarm Algorithm-Based Hybrid Fuzzy PD-PI Controller for Frequency Regulation of Distributed Power Generation System with Electric Vehicle. J. Control Autom. Electr. Syst. 2020, 31, 1-18. [CrossRef]

26. Alam, M.S.; Al-Ismail, F.S.; Abido, M.A. PV/Wind-Integrated Low-Inertia System Frequency Control: PSO-Optimized FractionalOrder PI-Based SMES Approach. Sustainability 2021, 13, 7622. [CrossRef]

27. Alam, M.S.; Al-Ismail, F.S.; Abido, M.A. Power management and state of charge restoration of direct current microgrid with improved voltage-shifting controller. J. Energy Storage 2021, 44, 103253. [CrossRef]

28. Jena, N.K.; Sahoo, S.; Nanda, A.B.; Sahu, B.K.; Mohanty, K.B. Frequency Regulation in an Islanded Microgrid with Optimal Fractional Order PID Controller. In Advances in Intelligent Computing and Communication; Springer: Berlin/Heidelberg, Germany, 2020; pp. 447-457.

29. Singh, A.; Suhag, S. Frequency regulation in an AC microgrid interconnected with thermal system employing multiverseoptimised fractional order-PID controller. Int. J. Sustain. Energy 2020, 39, 250-262. [CrossRef]

30. Satapathy, P.; Debnath, M.K.; Mohanty, P.K.; Sahu, B.K. Participation of Geothermal and Dish-Stirling Solar Power Plant for LFC Analysis Using Fractional-Order Controller. In Innovation in Electrical Power Engineering, Communication, and Computing Technology; Springer: Berlin/Heidelberg, Germany, 2020; pp. 113-122.

31. Saxena, S. Load frequency control strategy via fractional-order controller and reduced-order modeling. Int. J. Electr. Power Energy Syst. 2019, 104, 603-614. [CrossRef]

32. Babaei, F.; Lashkari, Z.B.; Safari, A.; Farrokhifar, M.; Salehi, J. Salp swarm algorithm-based fractional-order PID controller for LFC systems in the presence of delayed EV aggregators. IET Electr. Syst. Transp. 2020. [CrossRef]

33. Lamba, R.; Singla, S.K.; Sondhi, S. Design of Fractional Order PID Controller for Load Frequency Control in Perturbed Two Area Interconnected System. Electr. Power Components Syst. 2019, 47, 998-1011. [CrossRef]

34. Tian, E.; Peng, C. Memory-Based Event-Triggering $H_{\infty}$ Load Frequency Control for Power Systems Under Deception Attacks. IEEE Trans. Cybern. 2020. [CrossRef]

35. Kanagaraj, N. Photovoltaic and Thermoelectric Generator Combined Hybrid Energy System with an Enhanced Maximum Power Point Tracking Technique for Higher Energy Conversion Efficiency. Sustainability 2021, 13, 3144.

36. Kanagaraj, N.; Rezk, H. Dynamic Voltage Restorer Integrated with Photovoltaic-Thermoelectric Generator for Voltage Disturbances Compensation and Energy Saving in Three-Phase System. Sustainability 2021, 13, 3511. [CrossRef]

37. Mohammadzadeh, A.; Sabzalian, M.H.; Zhang, W. An interval type-3 fuzzy system and a new online fractional-order learning algorithm: Theory and practice. IEEE Trans. Fuzzy Syst. 2019. [CrossRef]

38. Arya, Y.; Kumar, N. Design and analysis of BFOA-optimized fuzzy PI/PID controller for AGC of multi-area traditional/restructured electrical power systems. Soft Comput. 2017, 21, 6435-6452. [CrossRef]

39. Meziane, K.B.; Naoual, R.; Boumhidi, I. Type-2 Fuzzy Logic based on PID controller for AGC of Two-Area with Three Source Power System including Advanced TCSC. Procedia Comput. Sci. 2019, 148, 455-464. [CrossRef]

40. Babahajiani, P.; Shafiee, Q.; Bevrani, H. Intelligent demand response contribution in frequency control of multi-area power systems. IEEE Trans. Smart Grid 2016, 9, 1282-1291. [CrossRef]

41. Boyd, S.P.; El Ghaoui, L.; Feron, E.; Balakrishnan, V. Linear Matrix Inequalities in System and Control Theory; SIAM: Philadelphia, PA, USA, 1994; Volume 15.

42. Xie, L. Output feedback H-infinity control of systems with parameter uncertainty. Int. J. Control 1996, 63, 741-750. [CrossRef]

43. Lu, J.G.; Chen, Y.Q. Robust stability and stabilization of fractional-order interval systems with the fractional order $\alpha$ : The $0 \leq \alpha \leq 1$ case. Autom. Control IEEE Trans. 2010, 55, 152-158. 